\title{
Quantum Kolmogorov-Sinai entropy and Pesin relation
}

\author{
Tomer Goldfriend and Jorge Kurchan \\ Laboratoire de Physique de l'Ecole Normale Supérieure, ENS, Université PSL, CNRS, Sorbonne Université, Université Paris-Diderot, \\ Sorbonne Paris Cité, 24 rue Lhomond, 75005 Paris, France
}

(Received 15 November 2020; accepted 3 June 2021; published 22 June 2021)

\begin{abstract}
We discuss a quantum Kolmogorov-Sinai entropy defined as the entropy production per unit time resulting from coupling the system to a weak, auxiliary bath. The expressions we obtain are fully quantum but require that the system is such that there is a separation between the Ehrenfest and the correlation timescales. We show that they reduce to the classical definition in the semiclassical limit, one instance where this separation holds. We show a quantum (Pesin) relation between this entropy and the sum of positive eigenvalues of a matrix describing phase-space expansion. Generalizations to the case where entropy grows sublinearly with time are possible.
\end{abstract}

DOI: 10.1103/PhysRevResearch.3.023234

\section{INTRODUCTION}

In classical mechanics the relations between thermalization, ergodicity, entropy, and chaos are well defined. The latter is characterized with the Lyapunov exponents and the Kolmogorov-Sinai (KS) entropy of trajectories in phase space. The analogous situation in closed quantum systems is less understood, e.g., the relation between entanglement entropy and thermalization [1], or entropy production and the quantum Lyapunov exponents. Previous studies expressed the entropy growth rate of a quantum system as the KS entropy of trajectories evolving in the underlying classical system [2-8], or which are obtained by projection [9]. Direct quantum generalizations of the KS entropy were also defined in the past by several rigorous mathematical derivations $[10,11]$ and, more recently, with a simple definition via a quantity conjectured as playing the role of the quantum Lyapunov spectrum, based on the out-of-time-order correlator (OTOC) [12].

In this paper we study a quantum KS entropy by weakly coupling the quantum system to an auxiliary bath-a setup which, in the classical case, gives a straightforward heuristic understanding of the equality between entropy production and the sum of positive Lyapunov exponents, the Pesin relation. The idea that in quantum systems, as in classical ones, the sensitivity of a system to an external weak noise can probe chaoticity goes back to Lindblad [13] (see also Refs. [3,14]). Here, we provide a general and simple definition, which is independent of a specific model and of the existence of a quantum Lyapunov regime. We derive explicitly the semiclassical limit, discuss quantum effects, and show how quantum Pesin-like relations exist when OTOCs grow exponentially.

The paper is organized as follows: In Sec. II we present a simple setup which yields the classical Pesin relation. The

Published by the American Physical Society under the terms of the Creative Commons Attribution 4.0 International license. Further distribution of this work must maintain attribution to the author $(s)$ and the published article's title, journal citation, and DOI. definition for the quantum Kolmogorov-Sinai entropy is introduced in Sec. III, where we obtain expression (28), which is our first general result. Then, in Sec. IV we analyze the semiclassical limit, and in Sec. V we derive a quantum Pesinlike relation, relating the KS entropy to the quantum Lyapunov exponents. This is given in expression (47), which is our second result. Its validity is more restricted than Eq. (28), because it assumes exponential growth of some quantities. In Sec. VI we discuss averaging over initial conditions and how to extend the result to other quantum setups. Finally, outlook and conclusion are given in Sec. VII.

\section{CLASSICAL PESIN RELATION FOR THE SIMPLE MINDED}

One way to introduce the Kolmogorov-Sinai entropy is as the entropy production around a trajectory, $\mathbf{x} \equiv(\mathbf{q}, \mathbf{p})$, of a system coupled to a weak auxiliary noise $\mathbf{h}(t)$ :

$$
\dot{x}^{\alpha}(t)=-\left\{H, x^{\alpha}\right\}+h^{\alpha}(t),
$$

with $H$ being the system's Hamiltonian, $\{\cdot, \cdot\}$ being the usual Poisson brackets, and the index $\alpha$ running over the components of a $2 N$ vector in phase space.

Consider a single classical, unperturbed trajectory. The Kolmogorov-Sinai entropies $h_{\mathrm{KS}}^{(q)}$ (for each $q$ ) for the ensemble of perturbations along this trajectory are defined as [15]

$$
t h_{\mathrm{KS}}^{(q)}=\frac{1}{1-q} \ln \left\{\int D[\text { history }] P^{q}[\text { history }]\right\},
$$

where the histories are all the trajectories starting from the same point $\mathbf{x}^{0}$ and ending in a point $\mathbf{x}^{f}[h]$ that depends on the noise realization. This definition may eventually be supplemented with averaging over initial conditions. We shall be interested in the limit in which the random perturbation is very weak.

Equation (2) is not the most easily generalizable to the quantum case, so we shall rewrite it as follows. First, we 
replicate $a=1, \ldots, q$

$$
\begin{aligned}
& \left\{\int D[\text { history }] P^{q}[\text { history }]\right\}=\int D[\mathbf{h}] P_{\text {ext }}[\mathbf{h}] \Pi_{a} \\
& \times \int_{\mathbf{x}_{a}=\mathbf{x}^{0}}^{\mathbf{x}^{f}} D\left[\mathbf{x}_{a}\right] \Pi_{\alpha} \delta\left[\dot{x}_{a}^{\alpha}(t)+\left\{H, x_{a}^{\alpha}\right\}-h^{\alpha}(t)\right],
\end{aligned}
$$

where the delta functions impose the trajectory equations and $P_{\text {ext }}[\mathbf{h}]$ is the distribution of the noise fields. Then, because these trajectories are casual, we may integrate away all the intermediate coordinates $\mathbf{x}_{a}$ except the last, to obtain

$$
\begin{aligned}
& \int D[\mathbf{h}] P_{\mathrm{ext}}[\mathbf{h}] \Pi_{a} \int_{\mathbf{x}_{a}=\mathbf{x}^{0}}^{\mathbf{x}^{f}} D\left[\mathbf{x}_{a}\right] \Pi_{\alpha} \delta\left(\dot{x}_{a}^{\alpha}(t)+\left\{H, x_{a}^{\alpha}\right\}-h^{\alpha}(t)\right) \\
& =\int D[\mathbf{h}] P_{\mathrm{ext}}[\mathbf{h}] \Pi_{a} \int d \mathbf{x}^{f} \delta\left(\mathbf{x}_{a}^{f}[h]-\mathbf{x}^{f}\right)=\int P^{q}\left(\mathbf{x}^{f}\right) d \mathbf{x}^{f} .
\end{aligned}
$$

What we have shown is that because of causality, integrals over powers of probabilities of trajectories may be traded for integrals of powers of probabilities over the (noise dependent) endpoint of the trajectories. In other words, we have gone from a description of "entropy of trajectories" to one of "production of the usual entropy at the end of the trajectory."

Starting from initial configurations within a small ball of radius $a$, and considering different noise realizations, we have at the end of the process at some time $t$ a "probability cloud." Its distribution is a function of the size of the initial set, the noise amplitude, and the stability properties of the trajectory. Denoting the probability distribution at time $t$ as $P(\mathbf{x}, t)$, we have the Rényi entropy of order $q$

$$
S_{q} \equiv \frac{1}{1-q} \ln \left\{\int d \mathbf{y} P^{q}(\mathbf{y}, t)\right\},
$$

and the corresponding Kolmogorov-Sinai entropy in terms of its exponential growth:

$$
h_{\mathrm{KS}}^{(q)} \equiv \frac{1}{t}\left[S_{q}(t)-S_{q}\left(t_{o}\right)\right],
$$

where $t_{o}$ is some short reference time. The advantage of this change of point of view is that it is easy to generalize the entropy production caused by weak noise to the quantum case.

Let us now proceed with the classical example. Consider the situation as in Fig. 1: In the absence of noise, in the directions that have exponential expansion between nearby trajectories, the displacement is $\Delta_{+} \sim a e^{\lambda^{\alpha}{ }_{t}}$, with $\lambda^{\alpha}$ being the positive Lyapunov exponents of the unperturbed system. In a Hamiltonian system, there will be exactly as many contracting modes that will compensate the effect of the expanding ones, $\Delta_{-} \sim a e^{-\lambda^{\alpha} t}$, and the volume of the set is unchanged. This is just Liouville's theorem and is a manifestation of the fact that without some form of coarse graining there is no entropy production in Hamiltonian dynamics. Let us consider now the effect of the noise: Roughly, expending and contracting directions are described by $\dot{\Delta}_{ \pm} \sim \pm \lambda^{\alpha} \Delta_{ \pm}+h(t)$, and the time evolution then gives $\Delta_{ \pm}(t)=\int_{0}^{t} e^{ \pm \lambda^{\alpha}\left(t-t^{\prime}\right)} h\left(t^{\prime}\right) d t^{\prime}$. One can see now that the integral which determines $\Delta_{+}$is dominated by initial times, $t^{\prime} \sim 0$, and the displacement induced by the noise will be amplified after a time $t$ as $\Delta_{+} \sim \epsilon e^{\lambda_{\alpha}^{+}} t$. However, now the contracting modes are compensated by the

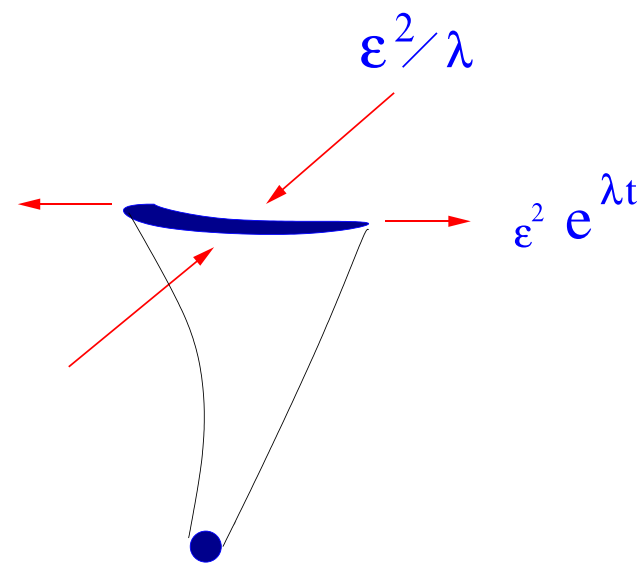

FIG. 1. The evolution of probability around a trajectory.

action of the noise at late times $t^{\prime} \sim t$, which gives a contribution $\Delta_{-} \sim \epsilon / \sqrt{\lambda_{\alpha}^{+}}$. Thus, if there are modes exponentially expanding in time, and we are interested in the phase-space volume $V=\Delta_{+} \Delta_{-}$of the "probability cloud" at the end of the process - up to logarithmic accuracy in time-we have $\ln V \sim t \sum_{\lambda_{i}>0} \lambda_{i}+O(\ln \epsilon)$. This is Pesin's relation. Let us see this in more detail.

\section{A. Preliminaries}

(i) "Democratic" dimensions. We consider a $2 N$ dimensional phase space ( $N$ coordinates and $N$ momenta). We normalize it with the canonical transformation $\tilde{x}=(b \mathbf{q}, \mathbf{p} / b)$, with $b$ being a parameter with the appropriate dimensions (and for simplicity from now on we drop the $\sim$ notation). Hereafter we take $b=\sqrt{m \omega}$, where $m$ and $\omega$ are the mass and frequency of an auxiliary oscillator Hamiltonian that will determine the initial state (see more details in the semiclassical derivation in Sec. IV below). Thus both position and momentum have units of $(\text { action })^{\frac{1}{2}}$.

(ii) White noise limit. The distribution of the noise fields $\mathbf{h}(t)$ is taken as

$$
P_{\mathrm{ext}}[\mathbf{h}(t)] \propto e^{-\frac{1}{2 \epsilon^{2} \tau} \sum_{\alpha} \int d t^{\prime} h^{\alpha}\left(t^{\prime}\right)^{2}},
$$

where $\tau$ is a typical timescale for the noise and $\epsilon$ has units of (energy/time $)^{\frac{1}{2}}$. That is, we can think of the random field as taken from a normal distribution $\mathcal{N}\left(0, \epsilon^{2}\right)$ independently at each time step $\tau$, or a Gaussian noise of correlation time $\tau$. Assuming that this timescale is shorter than any other relevant one in the system, we have the "white noise" limit, which is enough to yield the classical Pesin relation, even if the timescale $\tau$ does not disappear as a parameter of the problem. We shall assume the shortness of $\tau$ throughout the paper, also for the quantum problem. Note, however, that when doubt arises, one has to restore the noise correlations. In Sec. VII we discuss how the derivation can be generalized to account for a correlated noise.

\section{B. Entropy of trajectories' end points}

Deviations from a given trajectory can be described by the Poisson brackets (tangent space), which are given in terms of 
the second-derivative matrix:

$$
\begin{aligned}
R^{\alpha \gamma}\left(t, t^{\prime}\right) & \equiv \frac{\delta x^{\alpha}(t)}{\delta x^{\gamma}\left(t^{\prime}\right)}=\Omega^{\gamma \beta}\left\{x^{\alpha}(t), x^{\beta}\left(t^{\prime}\right)\right\} \\
& =\mathcal{T} \exp \left(-\int_{t^{\prime}}^{t} d t^{\prime} \Omega^{\gamma \delta}\left\{x^{\delta}(t),\left\{x^{\alpha}(t), \mathcal{H}\right\}\right\}\right),
\end{aligned}
$$

where we have introduced the $2 N \times 2 N$ matrix $\Omega=\left(\begin{array}{cc}0 & 1 \\ -1 & 0\end{array}\right)$ and $\mathcal{T}$ is the time-order operator. The last equality results from solving the tangent space dynamics for the Poisson brackets (see Appendix in Ref. [16]): $\frac{d}{d t}\left\{x^{\alpha}(t), x^{\beta}\left(t^{\prime}\right)\right\}=$ $\left\{x^{\gamma}(t),\left\{x^{\alpha}(t), \mathcal{H}\right\}\right\} \Omega^{\gamma \delta}\left\{x^{\delta}(t), x^{\beta}\left(t^{\prime}\right)\right\}$, with the initial condition $\left\{x^{\alpha}\left(t^{\prime}\right), x^{\beta}\left(t^{\prime}\right)\right\}=\Omega^{\alpha \beta}$.

The effect of the noise may be computed by considering the deviation it produces from a trajectory $y^{\alpha}(t) \equiv x^{\alpha}(t)-$ $x_{h=0}^{\alpha}(t)$. The tangent space dynamics, to first order in the noise amplitude, then reads

$$
y^{\alpha}(t)=\int_{0}^{t} d t^{\prime} R^{\alpha \beta}\left(t, t^{\prime}\right) h^{\beta}\left(t^{\prime}\right) .
$$

The $y^{\alpha}(t)$ are Gaussian variables with zero average, since they are sums of the $h^{\alpha}$. Let us now compute the distribution of the last point $P(\mathbf{y}, t)$, at the end of the process. We start with writing the distribution as a path integral over realizations of the noise, $P(\mathbf{y}, t)=$ $\int \Pi_{\alpha} \frac{\mathcal{D} h^{\alpha}}{\sqrt{2 \pi \epsilon^{2} \tau}} \delta\left[\mathbf{y}-\int_{0}^{t} d t^{\prime} \mathbf{R}\left(t, t^{\prime}\right) \mathbf{h}\left(t^{\prime}\right)\right] e^{-\frac{1}{2 \epsilon^{2} \tau} \sum_{\alpha} \int d t^{\prime} h^{\alpha}\left(t^{\prime}\right)^{2}}$.

Then, we Fourier-transform the delta function and solve the Gaussian integral (after completing the square) over the fields $\mathbf{h}$ to find

$$
\begin{aligned}
P(\mathbf{y}, t)= & \int \Pi_{\alpha} \frac{\mathcal{D} h^{\alpha}}{\sqrt{2 \pi \epsilon^{2} \tau}} d \hat{\mathbf{y}} \exp \left\{i \hat{\mathbf{y}}\left[\mathbf{y}-\int_{0}^{t} d t^{\prime} \mathbf{R}\left(t, t^{\prime}\right) \mathbf{h}\left(t^{\prime}\right)\right]\right. \\
& \left.-\frac{1}{2 \epsilon^{2} \tau} \sum_{\alpha} \int d t^{\prime} h^{\alpha}\left(t^{\prime}\right)^{2}\right\} \\
= & \int d \hat{\mathbf{y}} \exp \left\{i \mathbf{y} \hat{\mathbf{y}}-\frac{\epsilon^{2} \tau}{2} \int_{0}^{t} d t^{\prime} \hat{\mathbf{y}}^{T} \mathbf{R}\left(t, t^{\prime}\right) \mathbf{R}^{T}\left(t, t^{\prime}\right) \hat{\mathbf{y}}\right\} .
\end{aligned}
$$

The integral over the Fourier variable $\hat{\mathbf{y}}$ then gives

$$
\begin{aligned}
P(\mathbf{y}, t) & =\left(2 \pi \epsilon^{2} \tau\right)^{-d / 2}[\operatorname{det} \mathcal{A}]^{-\frac{1}{2}} e^{-\frac{1}{2 \epsilon^{2} \tau} \mathbf{y}^{T} \mathcal{A}^{-1} \mathbf{y}}, \\
\mathcal{A}^{\alpha \gamma}(t) & \equiv \sum_{\beta} \int_{0}^{t} d t^{\prime} R^{\gamma \beta}\left(t, t^{\prime}\right) R^{\alpha \beta}\left(t, t^{\prime}\right)=\int_{0}^{t} d t^{\prime} \mathcal{B}^{\alpha \gamma}\left(t, t^{\prime}\right) .
\end{aligned}
$$

Equation (11) thus describes how the weak noise spreads a single trajectory to a probability cloud whose distribution at time $t$ is Gaussian with a variance determined by the $2 N \times 2 N$ matrix $\mathcal{A}$-the main object here. We note that the matrix $\mathcal{B}\left(t, t^{\prime}\right)$ is symplectic, and it is the instantaneous contribution from time $t^{\prime}$ to the expansion at time $t$, while $\mathcal{A}(t)$ (which is generally not symplectic) is the total expansion. Taking the final expression for $P(\mathbf{y}, t)$, we can calculate the Rényi entropy and its exponential growth rate, according to Eqs. (5) and (6), respectively:

$$
e^{S_{2}(t)}=\frac{\mathcal{M}}{2} \operatorname{det}\left[\tau \epsilon^{2} \mathcal{A}(\mathbf{t})\right], \quad t h_{\mathrm{KS}}^{(2)}=\frac{1}{2} \ln \left(\frac{\operatorname{det} \mathcal{A}(t)}{\operatorname{det} \mathcal{A}\left(t_{o}\right)}\right),
$$

where $\mathcal{M}$ is an irrelevant constant and $t_{o}$ is a short reference time. For the moment we have not averaged over initial conditions.

\section{Pesin relation}

In the case in which the second-derivative matrix $\left\{x^{\delta},\left\{x^{\alpha}, \mathcal{H}\right\}\right\}$ is constant in time, one can easily confirm the claim above: If there is an exponential expansion in a mode of $\mathcal{B}$, then the initial times $t^{\prime} \sim 0$ dominate the time integral, and the expanding directions are of order $\epsilon e^{\lambda_{\alpha} t}$. Contracting modes of $\mathcal{B}$ are actually stopped from contracting completely by the action of the noise at late times $t^{\prime} \sim t$, which then give a contribution $\epsilon / \sqrt{\lambda_{\beta}}$. The general case is similar but slightly less trivial, as the tangent space vectors rotate in time. A rigorous approach is given by defining covariant Lyapunov vectors, as we explain now (a different derivation is also given in Appendix A 1).

The entropy production in Eq. (12) depends on the determinant of the matrix $\mathcal{A}$. First, we use the facts that $\mathbf{R}\left(t, t^{\prime}\right)=$ $\mathbf{R}(t, 0) \mathbf{R}\left(0, t^{\prime}\right)$ and $\mathbf{R}\left(0, t^{\prime}\right)=\mathbf{R}^{-1}\left(t^{\prime}, 0\right)$ to write

$$
\begin{aligned}
|\mathcal{A}(t)| & =\left|\int_{0}^{t} d t^{\prime} \mathbf{R}\left(t, t^{\prime}\right) \mathbf{R}^{T}\left(t, t^{\prime}\right)\right| \\
& =\left|\int_{0}^{t} d t^{\prime} \mathbf{R}(t, 0) \mathbf{R}^{-1}\left(t^{\prime}, 0\right)\left[\mathbf{R}^{T}\left(t^{\prime}, 0\right)\right]^{-1} \mathbf{R}^{T}(t, 0)\right| .
\end{aligned}
$$

Then, symplecticity of $\mathbf{R}$, namely, $\mathbf{R}^{-1}=\Omega^{-1} \mathbf{R}^{T} \Omega$, gives us

$$
\begin{aligned}
|\mathcal{A}(t)| & =\left|\int_{0}^{t} d t^{\prime} \mathbf{R}(t, 0) \Omega^{-1} \mathbf{R}^{T}\left(t^{\prime}, 0\right) \mathbf{R}\left(t^{\prime}, 0\right) \Omega \mathbf{R}^{T}(t, 0)\right| \\
& =\left|\mathbf{R}(t, 0) \Omega^{-1}\right|\left|\int_{0}^{t} d t^{\prime} \mathbf{R}^{T}\left(t^{\prime}, 0\right) \mathbf{R}\left(t^{\prime}, 0\right)\right|\left|\Omega \mathbf{R}^{T}(t, 0)\right| \\
& =\left|\mathbf{R}(t, 0) \Omega^{-1} \mathbf{R}^{T}(t, 0) \Omega\right|\left|\int_{0}^{t} d t^{\prime} \mathbf{R}^{T}\left(t^{\prime}, 0\right) \mathbf{R}\left(t^{\prime}, 0\right)\right| \\
& =\left|\int_{0}^{t} d t^{\prime} \mathbf{R}^{T}\left(t^{\prime}, 0\right) \mathbf{R}\left(t^{\prime}, 0\right)\right| .
\end{aligned}
$$

As a final step, we use the notion of covariant Lyapunov vectors [17-19]: For any time $t_{1}$ we have $2 N$ vectors ${ }^{1} \gamma_{k}\left(t_{1}\right)$ such that $\left\|\mathbf{R}\left(t^{\prime}+t_{1}, t_{1}\right) \gamma_{k}\left(t_{1}\right)\right\| \rightarrow \exp \left(\lambda_{k} t^{\prime}\right)$ as $t^{\prime} \rightarrow \infty$, with $\lambda_{k}$ being the Lyapunov exponent. Let us define the $2 N \times 2 N$ matrix $\Gamma(0)$ whose columns are the covariant Lyapunov vectors at time $t=0$ and write

$$
\begin{aligned}
|\mathcal{A}(t)| & =\left|\left[\Gamma(0) \Gamma^{T}(0)\right]^{-1}\right|\left|\Gamma^{T}(0) \mathcal{A}(t) \Gamma(0)\right| \\
& =\left|\left[\Gamma(0) \Gamma^{T}(0)\right]^{-1}\right|\left|\int_{0}^{t} d t^{\prime} \Gamma^{T}(0) \mathbf{R}^{T}\left(t^{\prime}, 0\right) \mathbf{R}\left(t^{\prime}, 0\right) \Gamma(0)\right| .
\end{aligned}
$$

\footnotetext{
${ }^{1}$ For simplicity we assume that there is no degeneracy and all Lyapunov exponents are distinct.
} 
Therefore, at times $t^{\prime}$ large enough within the integral, we have a matrix of the form $\mathcal{B}^{\beta \alpha}\left(t^{\prime}, 0\right) \sim v^{\alpha}\left(t^{\prime}\right) v^{\beta}\left(t^{\prime}\right) e^{\left(\lambda_{\alpha}+\lambda_{\beta}\right) t^{\prime}}$, leading to $|\mathcal{A}(t)| \rightarrow e^{\sum_{\lambda_{\alpha}>0} \lambda_{\alpha} t}$ at $t \rightarrow \infty$.

\section{Anticipating the quantum case}

We now go back to the classical result in Eq. (12). The left-hand side of this equation is also valid even in the absence of exponential divergences, although in that case the total entropy production does not scale linearly with time, and the KS entropy defined as a logarithmic entropy production per unit time goes to zero. This is also true for the quantum formula derived below. Two additional comments will be important for the quantum case:

(a) Coupling to the noise. Is it necessary to couple noise to all variables? The question is relevant classically because we usually couple noise only to the coordinates, and it appears in half of Hamilton's equations. It will be much more serious quantum mechanically, where there is considerably more freedom. Let us see the case when the noise is coupled to a single variable, say, $x^{1}$. We may redo the calculation above, to obtain a new matrix $\hat{\mathcal{A}}$ given by

$$
\hat{\mathcal{A}}^{\alpha \beta} \equiv \int d t^{\prime} R^{\alpha, 1}\left(t, t^{\prime}\right) R^{\beta, 1}\left(t, t^{\prime}\right)
$$

Once again, if we consider only the initial time $t^{\prime}=0$, the corresponding matrix will have rank 1 , and we shall obtain only one expansion direction. However, as we integrate over many $t^{\prime}$, provided the system is such that the eigenbasis is rotating with dynamics, we have that the rank of the matrix will increase as time passes. This applies to the "recent" times as well, which also have to be integrated so that they contribute fully to the contracting directions. We hence conclude that if at times $t$ for which the KS entropy is computed, the tangent space has rotated ergodically, then it is not important how many noises we have coupled to the system. We shall see below that quantum mechanically the situation is more subtle, and also more unavoidable. Let us note also that applying noise to a local (in space) operator, and seeing how entropy builds up, is an interesting question in itself.

(b) Initial distribution width. Suppose the initial condition $\mathbf{y}_{0}$, rather than being a single configuration, is spread over an isotropic Gaussian of width $a$ in phase space, as in Fig. 1. A simple modification of the steps leading to Eq. (11), namely, taking $\mathbf{y}=\int_{0}^{t} d t^{\prime} \mathbf{R}\left(t, t^{\prime}\right) \mathbf{h}\left(t^{\prime}\right)+\mathbf{R}(t, 0) \mathbf{y}_{0}$, gives

$S_{2}(t)-S_{2}(t=0)=\frac{1}{2} \ln \operatorname{det}\left[\epsilon^{2} \tau \mathcal{A}(t)+a^{2} \mathcal{B}(t, 0)\right]+$ const.

In the absence of noise, $\epsilon^{2} \mathcal{A}(t)=0$, and the right-hand side is a constant, because $\mathcal{B}$ is symplectic and has determinant 1 . Note that $a^{2}$, being the volume of a $(p, q)$ cell, has dimensions of action. We obtain the correct time dependence only if the expansion due to noise is large compared with the initial volume: $\frac{\epsilon^{2} \tau}{a^{2}} e^{\lambda t} \gg 1$. This gives us a warning of trouble ahead for the quantum case, where initial packet width is unavoidable and we are not allowed to take times to infinity.

\section{QUANTUM KOLMOGOROV-SINAI ENTROPY A DEFINITION}

Motivated by the classical case, we consider a quantum evolution coupling the system to a weak, auxiliary bath, which can be introduced from first principles as a coupling to an infinite set of oscillators, exactly the way one derives the classical Langevin equation [20]. We note that this line of reasoning was applied for understanding entropy production and decoherence in open quantum systems $[2,4,6]$.

If the temperature of the bath is infinite, friction may be neglected; otherwise, forward and backward propagations are with different fluctuating fields, as may be seen from the Schwinger-Keldysh formalism [21,22]. In this limit the bath becomes effectively classical. We can thus consider, for infinite auxiliary bath temperature, the Hamiltonian $H=H_{0}(\mathbf{x})+\sum_{\alpha} h^{\alpha}(t) x^{\alpha}(t)$, with the time-dependent random field $\mathbf{h}(t)$, which has probability distribution $P_{\text {ext }}[\mathbf{h}(t)]$ given in Eq. (7). For simplicity, as in the classical case, we shall consider that the correlation time of this bath $\tau$ is much smaller than the relevant timescales of the problem at hand (see discussion above concerning the white noise limit).

We are interested first in the Rényi entropy generated in the process, starting from an initial density matrix $\rho_{0}$. For the moment we restrict ourselves to $q=2$; in Appendix B 2 we generalize for all integer $q$. The expression for $S_{2}(t)$ involves a path integral over two different realizations of the noise, $\mathbf{h}_{1}$ and $\mathbf{h}_{2}$ :

$$
\begin{aligned}
& \operatorname{Tr}\left\{\rho^{2}(t)\right\} \\
& \quad=\operatorname{Tr}\left\{\int \mathcal{D} \mathbf{h}_{\mathbf{1}} \mathcal{D} \mathbf{h}_{\mathbf{2}} P_{\text {ext }}\left[\mathbf{h}_{\mathbf{1}}\right] P_{\text {ext }}\left[\mathbf{h}_{2}\right] U_{h_{2}, I}^{\dagger} U_{h_{1}, I} \rho_{0} U_{h_{1}, I}^{\dagger} U_{h_{2}, I} \rho_{0}\right\},
\end{aligned}
$$

where we work with the interaction picture $U_{h, I}=$ $\mathcal{T} \exp \left[-\frac{i}{\hbar} \int \sum_{\alpha} h^{\alpha}\left(t^{\prime}\right) x^{\alpha}\left(t^{\prime}\right) d t^{\prime}\right]$, with the operator $x(t)$ given in the Heisenberg picture of the unperturbed dynamics, $\mathbf{x}(t)=$ $e^{i H_{0} t / \hbar} \mathbf{x} e^{-i H_{0} t / \hbar}$

Our approach to evaluate Eq. (18) in the weak noise limit is as follows: We first approximate $\ln \operatorname{Tr} \rho^{2}(t)$ for a given random field, up to second order in $\mathbf{h}_{1,2}$, and then we average over the noise amplitude. The same procedure can be carried out for the analogous classical problem, which then reproduces the classical result in Eq. (17); this is shown explicitly in Appendix A 2. Let us then define

$$
\begin{aligned}
& \mathcal{Z}\left[\mathbf{h}_{\mathbf{1}}, \mathbf{h}_{\mathbf{2}}\right] \equiv \ln \operatorname{Tr}\left\{U_{h_{2}, I}^{\dagger} U_{h_{1}, I} \rho_{0} U_{h_{1}, I}^{\dagger} U_{h_{2}, I} \rho_{0}\right\}, \\
& \operatorname{Tr}\left\{\rho^{2}(t)\right\}=\int \mathcal{D} \mathbf{h}_{\mathbf{1}} \mathcal{D} \mathbf{h}_{\mathbf{2}} P_{\text {ext }}\left[\mathbf{h}_{\mathbf{1}}\right] P_{\text {ext }}\left[\mathbf{h}_{\mathbf{2}}\right] e^{\mathcal{Z}\left[\mathbf{h}_{\mathbf{1}}, \mathbf{h}_{\mathbf{2}}\right]},
\end{aligned}
$$

and perform time-dependent perturbation theory around $\mathbf{h}_{1}, \mathbf{h}_{2}=0$, up to second order. The first-order terms vanish, whereas the second-order terms depend only on the difference $\boldsymbol{\eta}=\mathbf{h}_{1}-\mathbf{h}_{2}$; we find (see derivation in Appendix B 1) 


$$
\operatorname{Tr} \rho^{2}(t) \approx \operatorname{Tr} \rho_{0}^{2} \int \mathcal{D} \eta P_{\text {ext }}[\eta] \exp \left(-\frac{1}{2} \sum_{\alpha, \beta} \int d t^{\prime} d t^{\prime \prime} \frac{\operatorname{Tr}\left\{i\left[x^{\alpha}\left(t^{\prime}\right), \rho_{0}\right] i\left[x^{\beta}\left(t^{\prime \prime}\right), \rho_{0}\right]\right\}}{\hbar^{2} \operatorname{Tr} \rho_{0}^{2}} \eta^{\alpha}\left(t^{\prime}\right) \eta^{\beta}\left(t^{\prime \prime}\right)\right) .
$$

We are left with solving Eq. (20) as a Gaussian integral over the fields $\eta^{\alpha}(t)$, recalling that, in the limit of small timescale $\tau$ of the bath, $P_{\text {ext }}[\eta] \propto e^{-\frac{1}{2 \epsilon^{2} \tau} \sum_{\alpha} \int d t^{\prime}\left[\eta^{\alpha}\left(t^{\prime}\right)\right]^{2}}$. Next, we obtain this solution by taking a simplified initial state $\rho_{0}$ and employing the matrix determinant lemma.

For simplicity we work hereafter with a Hilbert space which is represented by a real, orthogonal basis $\{|\mu\rangle\}$. Let us consider the case where the initial density matrix is given by a pure state, $\rho_{0}=\left|\phi_{0}\right\rangle\left\langle\phi_{0}\right|$. We shall generalize in Sec. VI to any initial condition. Developing the trace in the exponent of Eq. (20), we can write

$$
\operatorname{Tr}\left\{\left[x^{\alpha}\left(t^{\prime}\right), \rho_{0}\right]\left[x^{\beta}\left(t^{\prime \prime}\right), \rho_{0}\right]\right\}=\left\langle\phi_{0}\left|\left[x_{c}^{\alpha}\left(t^{\prime}\right) x_{c}^{\beta}\left(t^{\prime \prime}\right)+x_{c}^{\beta}\left(t^{\prime \prime}\right) x_{c}^{\alpha}\left(t^{\prime}\right)\right]\right| \phi_{0}\right\rangle,
$$

where

$$
x_{c}^{\alpha}(t) \equiv x^{\alpha}(t)-\left\langle\phi_{0}\left|x^{\alpha}(t)\right| \phi_{0}\right\rangle .
$$

We thus find

$$
\begin{aligned}
\operatorname{Tr} \rho^{2}(t) & \approx \int \mathcal{D} \boldsymbol{\eta} P[\boldsymbol{\eta}] e^{-\frac{1}{\hbar^{2}} \sum_{\mu}\left|\sum_{\alpha} \int_{0}^{t} d t^{\prime}\left\langle\phi_{0}\left|x_{c}^{\alpha}\left(t^{\prime}\right)\right| \mu\right\rangle \eta^{\alpha}\left(t^{\prime}\right)\right|^{2}} \\
& =\int \mathcal{D} \boldsymbol{\eta} P[\boldsymbol{\eta}] e^{-\frac{1}{\hbar^{2}} \sum_{\mu}\left(\left\{\sum_{\alpha} \int_{0}^{t} d t^{\prime}\left\langle\phi_{0}\left|x_{c}^{\alpha}\left(t^{\prime}\right)\right| \mu\right\rangle_{\mathrm{Re}} \eta^{\alpha}\left(t^{\prime}\right)\right\}^{2}+\left\{\sum_{\alpha} \int_{0}^{t} d t^{\prime}\left\langle\phi_{0}\left|x_{c}^{\alpha}\left(t^{\prime}\right)\right| \mu\right\rangle_{\operatorname{Im}} \eta^{\alpha}\left(t^{\prime}\right)\right\}^{2}\right)},
\end{aligned}
$$

where we recall that the wave functions $|\mu\rangle$ (and the noise) are real and the subscripts Re and Im refer to the real and imaginary parts of a complex number, respectively, e.g., $\left\langle\phi_{0}\left|x_{c}^{\alpha}\left(t^{\prime}\right)\right| \mu\right\rangle_{\operatorname{Re}} \equiv \operatorname{Re}\left(\left\langle\phi_{0}\left|x_{c}^{\alpha}\left(t^{\prime}\right)\right| \mu\right\rangle\right)$. We now simplify the solution to the Gaussian integral in Eq. (23) by using the matrix determinant lemma: For an invertible matrix $\mathbf{K}$ of order $k \times k$ and a matrix $\mathbf{U}$ of order $k \times m$ we have

$$
\operatorname{det}\left(\mathbf{K}+\mathbf{U} \mathbf{U}^{T}\right)=\operatorname{det}\left(\mathbf{I}_{m}+\mathbf{U}^{T} \mathbf{K}^{-1} \mathbf{U}\right) \operatorname{det}(\mathbf{K}),
$$

where $\mathbf{I}_{m}$ is the $m \times m$ identity matrix. If we now discretize time, $0=t_{1}<t_{2}<\cdots<t_{T}=t$, we can rewrite the path integral as an integral over a vector $\eta_{t_{n}}^{\alpha} \equiv \eta^{\alpha}\left(t_{n}\right)$ of length $2 N T$

$$
\operatorname{Tr} \rho^{2}(t)=\operatorname{det}\left|\frac{\mathbf{K}}{\epsilon^{2} \tau}\right|^{1 / 2} \int d \boldsymbol{\eta} \exp \left[-\frac{1}{2 \epsilon^{2} \tau} \eta_{t_{n}}^{\alpha} K_{t_{n} t_{m}}^{\alpha \beta} \eta_{t_{m}}^{\beta}-\frac{1}{2 \hbar^{2}} \eta_{t_{n}}^{\alpha}\left(U_{t_{n}}^{\alpha ; \mu} U_{t_{m}}^{\beta ; \mu}+V_{t_{n}}^{\alpha ; \mu} V_{t_{m}}^{\beta ; \mu}\right) \eta_{t_{m}}^{\beta}\right],
$$

where we define the $2 N T \times 2 N T$ matrix $K_{t_{n} t_{m}}^{\alpha \beta} \equiv \delta_{t_{n} t_{m}}^{\alpha \beta} d t$ and the $2 N T \times L$ matrices $U_{t_{n}}^{\alpha ; \mu} \equiv 2\left\langle\phi_{0}\left|x_{c}^{\alpha}\left(t_{n}\right)\right| \mu\right\rangle_{\operatorname{Re}} d t$ and $V_{t_{n}}^{\alpha ; \mu} \equiv$ $2\left\langle\phi_{0}\left|x_{c}^{\alpha}\left(t_{n}\right)\right| \mu\right\rangle_{\mathrm{Im}} d t$, with $L$ being the size of the Hilbert space and $N$ being the number of degrees of freedom. The solution to the Gaussian integral involves a determinant of a $2 N T \times 2 N T$ matrix, which, by employing the matrix determinant lemma, can be simplified to a determinant of a $2 L \times 2 L$ matrix:

$$
\begin{aligned}
\operatorname{Tr} \rho^{2}(t) & =\operatorname{det}\left|\frac{\mathbf{K}}{\epsilon^{2} \tau}\right|^{1 / 2} \operatorname{det}\left|\frac{\mathbf{K}}{\epsilon^{2} \tau}+\frac{1}{\hbar^{2}}\left(\begin{array}{ll}
\mathbf{U} & \mathbf{V}
\end{array}\right)\left(\begin{array}{c}
\mathbf{U}^{T} \\
\mathbf{V}^{T}
\end{array}\right)\right|^{-1 / 2} \\
& =\operatorname{det}\left|\mathbf{I}_{2 L}+\frac{\epsilon^{2} \tau}{\hbar^{2}}\left(\begin{array}{c}
\mathbf{U}^{T} \\
\mathbf{V}^{T}
\end{array}\right) \mathbf{K}^{-1}\left(\begin{array}{ll}
\mathbf{U} & \mathbf{V}
\end{array}\right)\right|^{-1 / 2} .
\end{aligned}
$$

Inserting back the definitions of $\mathbf{K}, \mathbf{U}$, and $\mathbf{V}$ and taking back the continuous time limit, we find

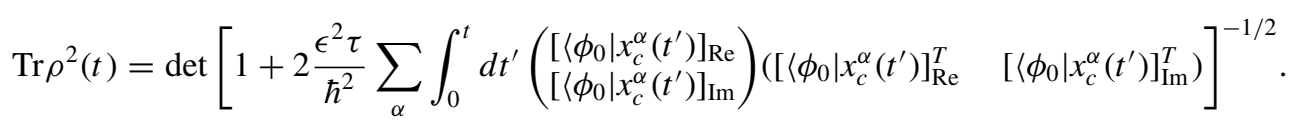

Note that the determinant is of a matrix that (i) has, at this stage, twice the dimension of Hilbert space $2 L \times 2 L$, and (ii) because it is a sum over tensor products, has positive eigenvalues. As a final step we may multiply the matrix within the determinant left and right with $\mathbf{Z} \equiv \frac{1}{\sqrt{2}}\left(\begin{array}{ll}\mathbf{I}_{L} & i_{\mathbf{I}_{L}} \\ -i \mathbf{I}_{L}\end{array}\right)$ and $\mathbf{Z}^{-1}$, respectively, to find

$$
\begin{gathered}
\operatorname{Tr} \rho^{2}(t)=\operatorname{det}\left[1+\frac{\epsilon^{2} \tau}{\hbar^{2}} \mathbf{A}\right]^{-1 / 2}=e^{-\left[S_{(2)}(t)-S_{(2)}(0)\right]}=e^{-t h_{\mathrm{KS}}^{(2)},} \\
\mathbf{A} \equiv \sum_{\alpha} \int_{0}^{t} d t^{\prime}\left(\begin{array}{cl}
x_{c}^{\alpha}\left(t^{\prime}\right)\left|\phi_{0}\right\rangle\left\langle\phi_{0}\right| x_{c}^{\alpha}\left(t^{\prime}\right) & x_{c}^{\alpha}\left(t^{\prime}\right)\left|\phi_{0}\right\rangle\left\langle\phi_{0}\right| x_{c}^{\alpha *}\left(t^{\prime}\right) \\
x_{c}^{\alpha *}\left(t^{\prime}\right)\left|\phi_{0}\right\rangle\left\langle\phi_{0}\right| x_{c}^{\alpha}\left(t^{\prime}\right) & x_{c}^{\alpha *}\left(t^{\prime}\right)\left|\phi_{0}\right\rangle\left\langle\phi_{0}\right| x_{c}^{\alpha *}\left(t^{\prime}\right)
\end{array}\right)=\sum_{\alpha} \int_{0}^{t} d t^{\prime}\left(\begin{array}{c}
x_{c}^{\alpha}\left(t^{\prime}\right)\left|\phi_{0}\right\rangle \\
x_{c}^{\alpha *}\left(t^{\prime}\right)\left|\phi_{0}\right\rangle
\end{array}\right) \otimes\left(\begin{array}{c}
\left\langle\phi_{0}\right| x_{c}^{\alpha}\left(t^{\prime}\right) \\
\left\langle\phi_{0}\right| x_{c}^{\alpha *}\left(t^{\prime}\right)
\end{array}\right),
\end{gathered}
$$

where we still assume that $\left|\phi_{0}\right\rangle$ and the Hilbert space bases are real.
Equation (28) with definition (29) is our first main result: A formula for the entropy production starting from $\left|\phi_{0}\right\rangle\left\langle\phi_{0}\right|$ 
due to a weak coupling to a bath. It is valid even when there is no exponential expansion, but if and when there is, we deduce a generalization to the classical Kolmogorov-Sinai entropy. In Appendix B 2 we repeat the calculation for a general Rényi entropy: The result is that these entropies differ when averaged over different starting situations ("multifractality," just as in the classical case), but the effect of quantum fluctuations alone is subdominant. There is no multifractality of quantum origin for a single trajectory.

A general comment is in order here: The fluctuating field is coupled only to the phase-space operators $x^{\alpha}$. However, as discussed at the end of Sec. II, this can be generalized easily to any coupling: If we consider a set of observables $\left\{\mathcal{O}^{\alpha}\right\}$ and the Hamiltonian $H(\mathbf{x})=H_{0}(\mathbf{x})+\sum_{\alpha} h^{\alpha}(t) \mathcal{O}^{\alpha}$, then expression (29) remains the same, with the $\left\{x_{c}^{\alpha}\right\}$ replaced by $\left\{\mathcal{O}_{c}^{\alpha}\right\}$. So far this situation is very much like the classical case; there is, however, a catch: Quantum mechanically we may in principle couple noises to operators that are not simple functions of the physical operators $x_{i}$, for example, the projector onto any specific wave function, so one could have an exponential number of independent noises applied to the system. We are not choosing to do this here; the noise terms are acting on physical observables and are in number of the same order as the degrees of freedom. This will have the important consequence that some final expressions may be written in lower-dimensional spaces.

For the rest of the paper we further discuss our quantum entropy production equation (28) and the KS entropy that it defines in the presence of an exponential growth.

\section{A. Additivity}

The KS entropy is a rate of entropy production. As such, our definition in Eq. (28) must admit the property of additivity. To verify this, let us consider two uncoupled systems in the Hilbert space $\mathcal{H}^{(1)} \otimes \mathcal{H}^{(2)}$ of size $L^{2}$. The matrix $\mathbf{A}$ in Eq. (29) is now constituted of terms of the form

$$
\begin{aligned}
& z_{c}^{(1)}\left[\left|\phi_{0}^{(1)}\right\rangle \otimes\left|\phi_{0}^{(2)}\right\rangle\right]\left[\left\langle\phi_{0}^{(1)}\right| \otimes\left\langle\phi_{0}^{(2)}\right|\right] \bar{z}_{c}^{(1)} \text { and } \\
& z_{c}^{(2)}\left[\left|\phi_{0}^{(1)}\right\rangle \otimes\left|\phi_{0}^{(2)}\right\rangle\right]\left[\left\langle\phi_{0}^{(1)}\right| \otimes\left\langle\phi_{0}^{(2)}\right|\right] \bar{z}_{c}^{(2)},
\end{aligned}
$$

where the superindices denote the space to which each $z$ belongs. These may be written as

$$
\begin{aligned}
& \left|\phi_{0}^{(2)}\right\rangle\left\langle\phi_{0}^{(2)}\left|\otimes z_{c}^{(1)}\right| \phi_{0}^{(1)}\right\rangle\left\langle\phi_{0}^{(1)}\right| \bar{z}_{c}^{(1)} \text { and } \\
& \left|\phi_{0}^{(1)}\right\rangle\left\langle\phi_{0}^{(1)}\left|\otimes z_{c}^{(2)}\right| \phi_{0}^{(2)}\right\rangle\left\langle\phi_{0}^{(2)}\right| \bar{z}_{c}^{(2)} \text {. }
\end{aligned}
$$

Consider now a basis for each space $\left\{\left|\phi_{0}\right\rangle,|\mu\rangle\right\}$, where the $|\mu\rangle$ are orthogonal to $\left|\phi_{0}\right\rangle$. In the tensor product of the two bases we have vectors such as (i) $\left|\phi_{0}\right\rangle \otimes\left|\phi_{0}\right\rangle$, (ii) $\left|\phi_{0}\right\rangle \otimes|\mu\rangle$, (iii) $|\mu\rangle \otimes\left|\phi_{0}\right\rangle$, and (iv) $|\mu\rangle \otimes|\mu\rangle$. Within subspaces (i) and (iv), all terms are zero. The left-hand-side terms of Eq. (31) are zero in subspace (ii) and equal to $z_{c}^{(1)}\left|\phi_{0}^{(1)}\right\rangle\left\langle\phi_{0}^{(1)}\right| \bar{z}_{c}^{(1)}$ in subspace (iii). Similarly, the right-hand-side terms of Eq. (31) are only nonzero in subspace (ii), where they take the value $z_{c}^{(2)}\left|\phi_{0}^{(2)}\right\rangle\left\langle\phi_{0}^{(2)}\right| \bar{z}_{c}^{(2)}$. The determinant of Eq. (29) is the determinant of a matrix with two different blocks, each of size $2 L-1 \times 2 L-1$, and the identity elsewhere. The determinant is then a product of the ones associated with each space, so that the logarithm is additive.

\section{B. Timescales}

We will check below in Sec. IV that our definition of the KS entropy gives the correct result in the semiclassical limit. However, for a truly quantum system, Eq. (29) seems problematic: If we let $\epsilon \rightarrow 0$ at finite $\hbar$, we will not get a finite limit for $h_{\mathrm{KS}}$ plus logarithmic corrections in $\epsilon$, but rather $\operatorname{Tr}\left\{\rho^{2}(t)\right\} \sim_{\epsilon \rightarrow 0} \frac{\epsilon^{2} \tau}{\hbar^{2}} \operatorname{Tr} A$. Yet, as we have seen when discussing additivity, the identity term within the determinant is essential to obtain a meaningful result, so we ought to understand its meaning. We have a dilemma: We need $\epsilon$ to be very small because we have assumed this in all our expansions, but we now see that in the limit $\epsilon \rightarrow 0$ the expression becomes meaningless. There is an apparently obvious way out of this: Choose times that are long enough, so that the exponential expansion of A compensates for the smallness of $\epsilon$. However, we expect there will be a limit to this: The Ehrenfest time at which a minimal quantum packet will expand throughout phase space.

We argue that this is an inherent problem, shared also by the very definition of quantum Lyapunov exponents themselves.

Let us take the dimensional factors away from the phase-space coordinates [which we had normalized to have dimensions of (action) $\left.\frac{1}{2}\right]$, writing $x^{\prime}=\sqrt{\frac{T}{\hbar}} x$ and defining $\mathbf{A}_{\mathbf{o}}$ as in Eq. (29) but with $x^{\prime}$, to get

$$
e^{-h_{\mathrm{KS}}^{(2)} t} \sim \operatorname{Tr}\left\{\rho^{2}(t)\right\}=\operatorname{det}\left|1+\frac{\epsilon^{2} \tau}{\hbar} A_{o}\right|^{-1 / 2} .
$$

We recognize that the second term has the interpretation of a phase-space expansion measured as the number of "quantum cell" sizes. Thus the comparison with the first term is just expressing the fact that expansion of a direction over a fraction of one quantum cell size does not contribute to the determinant.

Let us assume that there is, at least during some time, a Lyapunov expansion with a largest exponent $\lambda_{1}$, meaning that $\mathbf{A}_{\mathbf{o}}$ will have some elements growing as $\frac{1}{\lambda_{1}} e^{\lambda_{1} t}$. This expansion may last up to the Ehrenfest or scrambling time $t_{E}$, which we may estimate as

$$
e^{\lambda_{1} t_{E}} \sim \frac{k}{\hbar}
$$

where $k$ depends on the system, in particular, its number of degrees of freedom. (We note that its value is rarely specified in the literature: We shall not break with this tradition, but just specify that it has to be related to the unperturbed system and it does not have to involve $\epsilon^{2} \tau$.) Looking at (32), we conclude that we may hope to have a meaningful expression, valid at times just below the Ehrenfest time, if

$$
\frac{\epsilon^{2} \tau}{\lambda_{1} \hbar^{2}} k \gg 1
$$

It is now clear that the semiclassical limit, taken before $\epsilon \rightarrow 0$, is a possibility to have a well-defined KS entropy. However, there is more: There are cases when the constant $k$ is expected to scale as the number of degrees of freedom $k \sim N$, while the ordinary two-point correlation does not. For these kinds of systems, where there is a hierarchy between Ehrenfest or scrambling and correlation times (the reader will find a 
discussion of this in Ref. [23]), we may take $\epsilon \rightarrow 0$ with large $\epsilon^{2} \tau k$, and the Kolmogorov-Sinai entropy is well defined, with a nonzero value for it being possible.

\section{SEMICLASSICAL LIMIT}

Let us now evaluate our quantum definition for the KS entropy in the semiclassical limit. This can be done starting with the earlier expression of Eq. (20), as we show in Appendix A 3, or working with Eq. (29), as we show now. We need to evaluate the expectation value $\left\langle\phi_{0}\left|x_{c}^{\alpha}\left(t^{\prime}\right)\right| \mu\right\rangle$ in the semiclassical limit, where for simplicity of presentation we assume that the system evolves with the unperturbed Hamiltonian of the form $H_{0}(\mathbf{x})=\frac{\mathbf{p}^{2}}{2 m}+V(\mathbf{q})$. The unitary evolution in the semiclassical limit can be obtained in a standard way, developing the path integral around the classical trajectory: We have $\mathbf{x}=\mathbf{x}_{\mathrm{cl}}(t)+\mathbf{y}(t)$, with $\mathbf{x}_{\mathrm{cl}}(t)$ being the classical trajectory, and its quantum perturbation is described by the operator $\mathbf{y}=(\delta \mathbf{p}, \delta \mathbf{q})$ through the time-dependent Hamiltonian

$$
H_{\mathrm{sc}}(\mathbf{y})=\mathbf{y}^{T}\left(\begin{array}{cc}
\frac{1}{2 m} & 0 \\
0 & \frac{V^{\prime \prime}(t)}{2}
\end{array}\right) \mathbf{y},\left.\quad V^{\prime \prime}(t) \equiv \frac{\partial^{2} V}{\partial q^{\alpha} \partial q^{\beta}}\right|_{x_{\mathrm{cl}}(t)} .
$$

This is a harmonic oscillator with time-dependent frequencies: The evolution of $\mathbf{y}$ is linear, and it coincides with the classical counterpart. Therefore $y^{\alpha}(t)=R^{\alpha \beta}(t, 0) y_{0}^{\beta}$, with

$$
\mathbf{R}(t, 0) \equiv \mathcal{T} \exp \left[\int_{0}^{t} d t^{\prime}\left(\begin{array}{cc}
0 & \frac{1}{m} \\
-V^{\prime \prime}\left[\mathbf{x}_{\mathbf{c l}}(t)\right] & 0
\end{array}\right)\right] .
$$

Since, by construction, $\mathbf{x}_{\mathrm{cl}}$ is diagonal and $\left\langle\phi_{0}|\mathbf{y}| \phi_{0}\right\rangle=0$, we have $\left\langle\phi_{0}\left|x_{c}^{\alpha}\left(t^{\prime}\right)\right| \mu\right\rangle=\left\langle\phi_{0}\left|y^{\alpha}\left(t^{\prime}\right)\right| \mu\right\rangle$. The vectors which build the operator in Eq. (29) then read

$$
\left\langle\phi_{0}\right| x_{c}^{\alpha}\left(t^{\prime}\right)=R^{\alpha \beta}\left(t^{\prime}, 0\right)\left\langle\phi_{0}\right| y^{\beta}(0) .
$$

We now choose to work with a specific basis: The one of a set of $N$ harmonic oscillators of mass $m$ and frequency $\omega$. As described in the classical case, we normalize the operators such that they have the same dimensions, (action) $)^{\frac{1}{2}}$, taking $x=(b \mathbf{q}, \mathbf{p} / b)$ with $b=\sqrt{m \omega}$, which we shall assume in what follows. The oscillator Hamiltonian thus reads $H_{\text {ref }}=$ $\frac{\omega}{2} \sum_{a}\left[\left(p^{a}\right)^{2}+\left(q^{a}\right)^{2}\right]$. We take the ground state of this Hamiltonian to define the initial density $\left|\phi_{0}\right\rangle=|0\rangle$. This choice of basis and initial conditions is appropriate to obtain the semiclassical limit since the initial distribution is localized in phase space.

Let us now calculate the elements in Eq. (37), which gives the matrix $\mathbf{A}$ in the semiclassical limit. The Hilbert space breaks into subspaces of different total numbers of boson excitations of the oscillator Hamiltonian $H_{\text {ref }}$. The $y^{\alpha}(0)$, being linear, create, when acting on $\left|\phi_{0}\right\rangle$, states of one boson. Hence, in the space having two or more bosons, the matrix A is zero, and the determinant in Eq. (28) is determined by a $2 N$-dimensional matrix within the doubled one-boson subspace (recall that the dimensions of $\mathbf{A}$ are twice the size of the Hilbert space). This matrix is constructed by the $2 N \times$ $N$ blocks $W^{\alpha n} \equiv R^{\alpha \beta}\left(t^{\prime}, 0\right)\left\langle\phi_{0}\left|y^{\beta}(0)\right| 1^{n}\right\rangle=\sqrt{\frac{\hbar}{2}}\left[R^{\alpha q^{n}}\left(t^{\prime}, 0\right)-\right.$ $\left.i R^{\alpha p^{n}}\left(t^{\prime}, 0\right)\right]$, as

$$
\left.\mathbf{A}\right|_{\left\{|1\rangle^{N},|1\rangle^{N}\right\}} \underset{\hbar \rightarrow 0}{\longrightarrow}\left(\begin{array}{cc}
\mathbf{W}^{T} \mathbf{W} & \mathbf{W}^{T} \mathbf{W}^{*} \\
\mathbf{W}^{* T} \mathbf{W} & \mathbf{W}^{* T} \mathbf{W}^{*}
\end{array}\right)
$$

where $\left|1^{n}\right\rangle$ is the state of one boson for the $n$th degree of freedom and zero for all the others. Note the crucial role played by the identity in Eq. (28) outside this subspace. Since we are interested in the determinant, we can multiply the above matrix from left and right with $\tilde{\mathbf{Z}}^{-1} \equiv \frac{1}{\sqrt{2}}\left(\begin{array}{cc}\mathbf{I}_{L} & \mathbf{I}_{L} \\ i_{L} & -i \mathbf{I}_{L}\end{array}\right)$ and $\tilde{\mathbf{Z}}$, respectively, to find

$$
\left.A^{\alpha \gamma}\right|_{\left\{|1\rangle^{N},|1\rangle^{N}\right\}} \underset{\hbar \rightarrow 0}{\longrightarrow} \sum_{\alpha} \int d t^{\prime}\left(\begin{array}{ll}
R^{\alpha q^{\beta}}\left(t^{\prime}, 0\right) R^{\alpha q^{\gamma}}\left(t^{\prime}, 0\right) & R^{\alpha p^{\beta}}\left(t^{\prime}, 0\right) R^{\alpha p^{\gamma}}\left(t^{\prime}, 0\right) \\
R^{\alpha p^{\beta}}\left(t^{\prime}, 0\right) R^{\alpha q^{\gamma}}\left(t^{\prime}, 0\right) & R^{\alpha p^{\beta}}\left(t^{\prime}, 0\right) R^{\alpha p^{\gamma}}\left(t^{\prime}, 0\right)
\end{array}\right) .
$$

We thus show that in the semiclassical limit the second Rényi entropy is given by

$$
\ln \operatorname{Tr}\left\{\rho^{2}(t)\right\}=\frac{1}{2} \ln \operatorname{det}\left[1+\frac{\epsilon^{2} \tau}{\hbar} \int_{0}^{t} d t^{\prime} \mathbf{R}^{T}\left(t^{\prime}, 0\right) \mathbf{R}\left(t^{\prime}, 0\right)\right] .
$$

As a final step, we can take, inversely, the steps which bring us from Eq. (13) to Eq. (14), employing the symplecticity of R. We multiply Eq. (40) from left and right with $\mathbf{R}(t, 0) \Omega^{-1}$ and $\Omega \mathbf{R}^{T}(t, 0)$, respectively, to obtain

$$
\begin{aligned}
S_{2}(t)-S_{2}(t=0) & =\ln \operatorname{Tr}\left\{\rho^{2}(t)\right\} \\
& =\frac{1}{2} \ln \operatorname{det}\left[\mathcal{B}(t, 0)+\frac{\epsilon^{2} \tau}{\hbar} \mathcal{A}(t)\right]
\end{aligned}
$$

This is to be compared with the classical expression (17), where the quantum initial state plays the role of the initial packet, with $a^{2} \leftrightarrow \hbar$.

If we now let $\hbar \rightarrow 0$ before $\epsilon \rightarrow 0$, we are in the same situation as the limit of small size $a$ of the initial packet in the classical case. If, on the contrary, we make $\hbar \rightarrow 0$ after $\epsilon \rightarrow 0$, the result is not the Lyapunov expansion.

\section{QUANTUM PESIN RELATIONS}

Several quantum generalizations of the KS entropy were suggested previously [9-12]. In particular, Ref. [12] defined an entropy via quantum Lyapunov exponents, which are in turn defined by the out-of-time-order commutator (OTOC) [24]: The square of a commutator of two operators at different times, $\left[\mathcal{O}^{\alpha}(t), \mathcal{O}^{\beta}(0)\right]^{2}$. We now make a connection between this notion and our formula (29).

Let us split A into early and late times as follows:

$$
\begin{aligned}
& \mathbf{A}^{-} \equiv \sum_{\alpha} \int_{0}^{\bar{t}} d t^{\prime}\left(\begin{array}{c}
\left\langle\phi_{0}\right| x_{c}^{\alpha}\left(t^{\prime}\right) \\
\left\langle\phi_{0}\right| x_{c}^{\alpha *}\left(t^{\prime}\right)
\end{array}\right)\left(\left\langle\phi_{0}\right| x_{c}^{\alpha}\left(t^{\prime}\right) \quad\left\langle\phi_{0}\right| x_{c}^{\alpha *}\left(t^{\prime}\right)\right), \\
& \mathbf{A}^{+} \equiv \sum_{\alpha} \int_{\bar{t}}^{t} d t^{\prime}\left(\begin{array}{c}
\left\langle\phi_{0}\right| x_{c}^{\alpha}\left(t^{\prime}\right) \\
\left\langle\phi_{0}\right| x_{c}^{\alpha *}\left(t^{\prime}\right)
\end{array}\right)\left(\left\langle\phi_{0}\right| x_{c}^{\alpha}\left(t^{\prime}\right) \quad\left\langle\phi_{0}\right| x_{c}^{\alpha *}\left(t^{\prime}\right)\right),
\end{aligned}
$$


such that

$$
t h_{\mathrm{KS}}^{(2)}=\frac{1}{2} \ln \operatorname{det}\left[1+\frac{\epsilon^{2} \tau}{\hbar^{2}} \mathbf{A}^{+}+\frac{\epsilon^{2} \tau}{\hbar^{2}} \mathbf{A}^{-}\right] .
$$

Now, because both $\mathbf{A}^{+}+1$ and $\mathbf{A}^{-}$are positive semidefinite, we may apply Minkowski's determinant inequality:

$$
\begin{aligned}
\operatorname{det}\left[1+\frac{\epsilon^{2} \tau}{\hbar^{2}} \mathbf{A}^{+}+\frac{\epsilon^{2} \tau}{\hbar^{2}} \mathbf{A}^{-}\right]^{1 / 2 L} \geqslant & \operatorname{det}\left[1+\frac{\epsilon^{2} \tau}{\hbar^{2}} \mathbf{A}^{+}\right]^{1 / 2 L} \\
& +\operatorname{det}\left[\frac{\epsilon^{2} \tau}{\hbar^{2}} \mathbf{A}^{-}\right]^{1 / 2 L}
\end{aligned}
$$

which gives

$$
\ln \operatorname{det}\left[1+\frac{\epsilon^{2} \tau}{\hbar^{2}} \mathbf{A}^{+}+\frac{\epsilon^{2} \tau}{\hbar^{2}} \mathbf{A}^{-}\right] \geqslant \ln \operatorname{det}\left[1+\frac{\epsilon^{2} \tau}{\hbar^{2}} \mathbf{A}^{+}\right] \text {. }
$$

Let us use this inequality to find a lower bound $\bar{h}_{\mathrm{KS}}^{(2)} \leqslant h_{\mathrm{KS}}^{(2)}$, which we shall later argue may be exact in some cases. We proceed as follows: We choose the time $\bar{t}$ such that for $\bar{t}<$ $t^{\prime}<t$ we may consider that $\mathbf{A}^{+}\left(t^{\prime}\right) \sim \mathbf{A}^{+}(t)$. The time $\bar{\tau} \equiv$ $t-\bar{t}$ is of the order of the autocorrelation of the $x^{\alpha}$. Then, we define $\bar{h}_{\mathrm{KS}}^{(2)}$ with

$$
\begin{aligned}
\bar{h}_{\mathrm{KS}}^{(2)} t \sim & -\ln \operatorname{det} \mid 1+\frac{\bar{\tau} \epsilon^{2} \tau}{\hbar^{2}} \sum_{\alpha}\left(\begin{array}{c}
x_{c}^{\alpha}(t)\left|\phi_{0}\right\rangle \\
x_{c}^{\alpha *}(t)\left|\phi_{0}\right\rangle
\end{array}\right) \\
& \left.\otimes\left(\begin{array}{c}
\left\langle\phi_{0}\right| x_{c}^{\alpha}(t) \\
\left\langle\phi_{0}\right| x_{c}^{\alpha *}(t)
\end{array}\right)\right|^{-1 / 2} .
\end{aligned}
$$

We can then use the matrix determinant lemma [Eq. (24)] to obtain, under these assumptions, an expression in terms of a $2 N \times 2 N$ determinant: $\bar{h}_{\mathrm{KS}}^{(2)} t=\frac{1}{2} \ln \operatorname{det} \mid \delta_{\alpha \beta}+$ $\frac{\bar{\tau} \epsilon^{2} \tau}{\hbar^{2}}\left\langle\phi_{0}\left|x_{c}^{\alpha}(t) x_{c}^{\beta}(t)+x_{c}^{\alpha *}(t) x_{c}^{\beta *}(t)\right| \phi_{0}\right\rangle \mid$, which gives

$$
\begin{aligned}
\bar{h}_{\mathrm{KS}}^{(2)} t & =\frac{1}{2} \ln \operatorname{det}\left|\delta_{\alpha \beta}+\frac{\bar{\tau} \epsilon^{2} \tau}{\hbar^{2}}\left\langle\phi_{0}\left|x_{c}^{\alpha}(t) x_{c}^{\beta}(t)+x_{c}^{\beta}(t) x_{c}^{\alpha}(t)\right| \phi_{0}\right\rangle\right| \\
& =\frac{1}{2} \ln \operatorname{det}\left|\delta_{\alpha \beta}+\frac{\bar{\tau} \epsilon^{2} \tau}{\hbar^{2}}\left\langle\phi_{0}\left|\left[x^{\alpha}(t), \rho_{0}\right]\left[x^{\beta}(t), \rho_{0}\right]\right| \phi_{0}\right\rangle\right|,
\end{aligned}
$$

where we have used the relation in Eq. (21) and the Hermiticity of the $x^{\alpha}$ and have assumed that the Hilbert space is given by real eigenfunctions. Equation (47) is in line with previous works which showed a relation between the second Rényi entropy and the OTOC, where the operator at the initial time appearing in the commutator can be taken as the density matrix [25-27].

Now the determinants which appear in Eq. (47) are of $2 N \times 2 N$ matrices. If there are exponential expansions and contractions of the $2 N \times 2 N$ matrix

$$
\begin{aligned}
\hat{\mathbf{A}}_{\alpha \beta} & \equiv\left\langle\phi_{0}\left|x_{c}^{\alpha}(t) x_{c}^{\beta}(t)+x_{c}^{\beta}(t) x_{c}^{\alpha}(t)\right| \phi_{0}\right\rangle \\
& =\left\langle\phi_{0}\left|\left[x^{\alpha}(t), \rho_{0}\right]\left[x^{\beta}(t), \rho_{0}\right]\right| \phi_{0}\right\rangle,
\end{aligned}
$$

with eigenvalues $e^{\lambda_{i} t}$, then thanks to the $\delta_{\alpha \beta}$ term we have

$$
\bar{h}_{\mathrm{KS}}^{(2)}=\sum_{\lambda_{i}>0} \lambda_{i} .
$$

Note the somewhat optimistic notation: We have not shown that the $\lambda_{i}$ deserve to be called individually "Lyapunov exponents," although it is very tempting to interpret them this way. Indeed, if there are exponential expansions, we may expect the inequality to be saturated, and $\bar{h}_{\mathrm{KS}}^{(2)}=h_{\mathrm{KS}}^{(2)}$ : This is because the smallness of the time interval $\bar{\tau}$ is compensated by the exponential expansion. We shall check this in the semiclassical limit.

A similar relation of the form $h_{\mathrm{KS}}^{(2)}=\sum_{\lambda_{i}>0} \lambda_{i}$ was already proposed in Ref. [12] as a definition of the quantum KS entropy, where the $\lambda_{i}$ are defined instead on the basis of the eigenvalues of the matrix $\mathbf{L}^{\alpha \beta}(t)=$ $-\left\langle\psi\left|\left[x^{\alpha}(t), x^{\gamma}(0)\right]\left[x^{\gamma}(t), x^{\beta}(0)\right]\right| \psi\right\rangle$, with $|\psi\rangle$ being an eigenstate of the Hamiltonian. (Another different definition of the Lyapunov spectrum was given in Ref. [28].) Let us insist that the relation we derive here is not a definition, but rather a derivation in terms of the entropy production.

\section{Check of the semiclassical limit}

One can check the semiclassical limit on the second equality of Eq. (47): As shown in Sec. IV, we have $x_{c}^{\alpha}(t) \underset{\hbar \rightarrow 0}{\longrightarrow}$ $R^{\alpha \gamma}(t, 0) y^{\gamma}(0)$; thus

$$
\begin{aligned}
& \left\langle\phi_{0}\left|x_{c}^{\alpha}(t) x_{c}^{\beta}(t)+x_{c}^{\beta}(t) x_{c}^{\alpha}(t)\right| \phi_{0}\right\rangle \\
& \quad=\sum_{\gamma \delta} R^{\alpha \gamma}(t, 0) R^{\beta \delta}(t, 0)\left\langle\phi_{0}\left|y^{\gamma}(0) y^{\delta}(0)+y^{\delta}(0) y^{\gamma}(0)\right| \phi_{0}\right\rangle .
\end{aligned}
$$

The matrix $\left\langle\phi_{0}\left|y^{\gamma}(0) y^{\delta}(0)+y^{\delta}(0) y^{\gamma}(0)\right| \phi_{0}\right\rangle$ is simply the identity matrix times $\hbar$ : Recall that for the semiclassical limit we choose $\left|\phi_{0}\right\rangle$ as the oscillator vacuum; therefore, if the indices correspond to different degrees of freedom, the expectation breaks into a product of two expectations, both vanishing, whereas if they correspond to a $p^{a}$ and its partner $q^{a}$, they also vanish, and $\left\langle\phi_{0}\left|\left[y^{\gamma}(0)\right]^{2}\right| \phi_{0}\right\rangle=\hbar$. Then,

$$
\begin{aligned}
\bar{h}_{\mathrm{KS}}^{(2)} t & \underset{\hbar \rightarrow 0}{\longrightarrow} \ln \operatorname{det}\left|1+\frac{\bar{\tau} \epsilon^{2} \tau}{\hbar} R(t, 0) R^{T}(t, 0)\right|^{-1 / 2} \\
& =\frac{1}{2} \operatorname{det}\left|1+\frac{\bar{\tau} \epsilon^{2} \tau}{\hbar} \mathcal{B}(t, 0)\right|
\end{aligned}
$$

which is the correct classical result, and thus $\bar{h}_{\mathrm{KS}}^{(2)}=h_{\mathrm{KS}}^{(2)}$ in this case.

\section{AVERAGING OVER INITIAL STATES}

In the analysis above we have assumed that (i) the Hilbert space is represented by real and orthogonal eigenfunctions $|\mu\rangle$ and (ii) the initial state is given by one of these eigenfunctions $\rho_{0}=\left|\phi_{0}\right\rangle\left\langle\phi_{0}\right|$. These assumptions facilitate the derivation of the KS entropy in Eq. (28) and were suitable for taking its semiclassical limit. We now discuss how to generalize the result beyond these assumptions.

Concerning point (i), it is important to extend the result to a nonreal eigenbasis since the derivation, as well as the proof of additivity, assumes a basis which is orthonormal to $\left|\phi_{0}\right\rangle$. We can always construct such a basis; however, depending on $\left|\phi_{0}\right\rangle$, it might not be real. The derivation can be readily extended to any choice of eigenbasis $\{|\mu\rangle\}$. The assumption 
of real eigenbasis was only to facilitate the notations for $\left[\left\langle\phi_{0}\right| x^{\alpha}\left(t^{\prime}\right)\right]^{*}=\left\langle\phi_{0}\right| x^{\alpha *}\left(t^{\prime}\right)$. For the general case, the matrix $\mathbf{A}$, which is constructed by four blocks, reads

$$
\mathbf{A}=\sum_{\alpha} \int_{0}^{t} d t^{\prime}\left(\begin{array}{rl}
\left\langle\mu\left|x_{c}^{\alpha}\left(t^{\prime}\right)\right| \phi_{0}\right\rangle\left\langle\phi_{0}\left|x_{c}^{\alpha}\left(t^{\prime}\right)\right| v\right\rangle & \left\langle\mu\left|x_{c}^{\alpha}\left(t^{\prime}\right)\right| \phi_{0}\right\rangle\left\langle\phi_{0}\left|x_{c}^{\alpha}\left(t^{\prime}\right)\right| v\right\rangle^{*} \\
\left\langle\mu\left|x_{c}^{\alpha}\left(t^{\prime}\right)\right| \phi_{0}\right\rangle^{*}\left\langle\phi_{0}\left|x_{c}^{\alpha}\left(t^{\prime}\right)\right| v\right\rangle & \left\langle\mu\left|x_{c}^{\alpha}\left(t^{\prime}\right)\right| \phi_{0}\right\rangle^{*}\left\langle\phi_{0}\left|x_{c}^{\alpha}\left(t^{\prime}\right)\right| v\right\rangle^{*}
\end{array}\right) .
$$

We are left with relaxing assumption (ii): Let us now show how we can average over initial conditions, generalizing the result beyond the case of $\rho_{0}=\left|\phi_{0}\right\rangle\left\langle\phi_{0}\right|$. The state $\left|\phi_{0}\right\rangle$ may be chosen as the ground state of an oscillator, translated by $\left(q_{o}^{i}, p_{o}^{i}\right)$. In other words, $\left|\phi_{0}\right\rangle$ is the coherent state $|z\rangle$ :

$$
\left|\phi_{0}\right\rangle=e^{i q_{o}^{i} p_{i}-i p_{o}^{i} q_{i}}|0\rangle=e^{z_{i} \alpha_{i}^{\dagger}-z_{i}^{*} \alpha_{i}}|0\rangle=|\mathbf{z}\rangle,
$$

where $z^{a}=q_{o}^{a}+i p_{o}^{a}$ and $\alpha_{a}^{\dagger}=q_{a}-i p_{a}$.

This immediately suggests how to average over initial conditions. Consider, for example, theGibbs-Boltzmann measure $\rho_{\mathrm{GB}}$. Like any operator, it may be expressed in its $P$-symbol representation [29]

$$
\rho_{\mathrm{GB}}=\int d z d \bar{z} \rho_{\mathrm{GB}}^{P}(z, \bar{z})|z\rangle\langle z|,
$$

where $\rho_{\mathrm{GB}}^{P}(z, \bar{z})$ is a phase-space function representing $\rho_{\mathrm{GB}}$. Then, a Rényi entropy averaged over the Gibbs-Boltzmann entropy reads

$$
\left\langle e^{h_{\mathrm{KS}}^{(2)}}\right\rangle_{\mathrm{GB}}=-\int d z d \bar{z} \rho_{\mathrm{GB}}^{P}(z, \bar{z}) \operatorname{Tr}_{z}\left\{\rho^{2}(t)\right\}
$$

and

$$
\operatorname{Tr}_{z}\left\{\rho^{2}(t)\right\}=\operatorname{det}\left|1+\frac{\epsilon^{2} \tau}{2 \hbar^{2}} \mathbf{A}_{z}\right|^{-1 / 2},
$$

with $\mathbf{A}_{\mathbf{z}}$ defined as $\mathbf{A}$ in Eq. (29), replacing $\left|\phi_{0}\right\rangle$ with $|z\rangle$. The same can obviously be done for all Rényi entropies $S_{q}$. Note that it is in this averaging over $z$ that the nontrivial dependence on $q$ ("multifractality") enters.

\section{Other groups, localized initial state}

Writing the Rényi entropy with averaging over coherent states, the generalization to spin systems seems natural. Suppose we have a spin- $j$ chain. We construct the coherent states for each site in this representation in the standard way:

$$
|z\rangle=\Pi_{i}\left|z_{i}\right\rangle=e^{\sum_{i} z_{i} J_{i}^{+}}|0\rangle ; \quad J_{i}^{-}|0\rangle=0 .
$$

Next, we consider two independent operators per site, for example, $J_{i}^{x}$ and $J_{i}^{y}$, and construct in this way the corresponding operator $A=\int^{i} d t^{\prime} \sum_{i}^{\alpha} J_{i}^{\alpha}\left(t^{\prime}\right)|z\rangle\langle z| J_{i}^{\alpha}\left(t^{\prime}\right)$, where $\alpha=x, y$. All the previous steps follow, including the semiclassical limit for $j \rightarrow \infty$.

For fermion systems, a similar strategy may be envisaged, replacing the spin operators by bifermionic operators $a_{i}^{\dagger} a_{j}$.

\section{SUMMARY AND OUTLOOK}

We have discussed a Kolmogorov-Sinai entropy of a closed quantum system by coupling it weakly to an auxiliary bath. The construction here allows one to derive (rather than assume) a quantum version of the Pesin relation. The external bath is classical in the sense that it is Markovian. However, the same steps of the analysis may be generalized to a truly quantum bath with a finite temperature and friction through the Schwinger-Keldysh formalism. We note that adding noise correlation can simply be done through the matrix $\mathbf{K}$ in Eq. (25). However, the case of friction is less trivial, since within the Schwinger-Keldysh formalism, it means that forward and backward trajectories are not identical, and the second Rényi entropy in Eq. (18) will thus involve four realizations of the noise.

In this paper we considered the instability generated in the system by the bath when coupled to a set of operators that is of the order of the number of degrees of freedom, or a subset of them. This has the important consequence that some final expressions may be written in lower-dimensional spaces. One could of course have used a much larger set of operators, including projectors onto pure states, and the result would have probably been different. Our procedure is thus an arbitrary, if physically motivated, choice.

A possibility that is physically interesting is to couple noise to a set of operators that are local in real space. As noted above, definition (28) is not restricted to systems where there is an exponential growth of OTOC-like quantities, and some, though not all, of the results in this paper carry through when there are no exponential growths in time, as is believed to be the case in local spin systems.

It would also be interesting to establish contact with the mathematical literature on the subject, in particular, with the Connes-Narnhofer-Thirring entropy [10]. Note that this entropy is defined in the thermodynamic limit and thus connection between the two definitions shall be done in view of the macroscopic limit.

The recent bound on the quantum Lyapunov exponent states that the exponential growth rate of the OTOC cannot be larger than $2 \pi k_{B} T / \hbar$, with $T$ being the temperature [23]. This result has simulated an enormous body of theoretical and experimental studies in various fields. An interesting future direction would be to study a quantum bound on the $\mathrm{KS}$ entropy. Indeed, it is natural to have a temperature-dependent bound on the growth rate of an entropy, which, unlike the Lyapunov exponent, is an extensive thermodynamic quantity.

Finally, it would be interesting to understand whether the production rate of entanglement entropy in a closed quantum system, which might be driving thermalization [1], follows the KS entropy. Several works have already established such a relation in semiclassical setups [5-8] or by projecting the quantum unitary evolution to an effective semiclassical one [9].

\section{ACKNOWLEDGMENT}

T.G. and J.K. are supported by Simons Foundation Grant No. 454943. 


\section{APPENDIX A: CLASSICAL DERIVATION}

In this Appendix we provide (i) rigorous grounds for the classical Pesin relation given in Sec. II, (ii) a classical derivation involving Poisson brackets, which is completely analog to the quantum one, and (iii) an alternative semiclassical derivation.

\section{Classical Pesin relation: More details}

In order to see how contributions from the noise at different times add, the natural objects to consider are the $p$ forms $\Lambda_{i_{1}, \ldots, i_{p}}(t)$, which represent the volume elements of dimension $p$. These expand according to [30] $\Sigma_{i_{1}, \ldots, i_{p}} \Lambda_{i_{1}, \ldots, i_{p}}^{2}(t) \sim$ $e^{t \Lambda^{(p)}} \Sigma_{i_{1}, \ldots, i_{p}} \Lambda_{i_{1}, \ldots, i_{p}}^{2}(0) \sim e^{\left(\lambda_{1}+\cdots+\lambda_{p}\right) t} \Sigma_{i_{1}, \ldots, i_{p}} \Lambda_{i_{1}, \ldots, i_{p}}^{2}(0)$. For a physicist, the easiest way to represent this is introducing fermions $a_{i}, a_{i}^{\dagger}$ and their vacuum state $|0\rangle$ and writing the contribution from all the $t^{\prime}$ as [31,32]

$$
\begin{aligned}
\Lambda_{i_{1}, \ldots, i_{p}}(t)= & \int d t^{\prime}\langle 0| a_{i_{1}} \cdots a_{i_{p}}\left[R_{i_{i} l_{1}}\left(t, t^{\prime}\right) a_{l_{1}}^{\dagger}\right] \\
& \cdots\left[R_{i_{p} l_{p}}\left(t, t^{\prime}\right) a_{l_{p}}^{\dagger}\right]|0\rangle .
\end{aligned}
$$

In fact, introducing another family of fermions $b_{i}, b_{i}^{\dagger}$ and the vacuum of both species, we have

$$
\begin{aligned}
& \Sigma_{i_{1}, \ldots, i_{p}} \Lambda_{i_{1}, \ldots, i_{p}}^{2}(t)=\Sigma_{i_{1}, \ldots, i_{p}} \int d t^{\prime} \\
& \left\langle 0\left|a_{i_{1}} \cdots a_{i_{p}} \cdots b_{i_{1}} \cdots b_{i_{p}}\left[R_{i_{i} m_{1}}\left(t, t^{\prime}\right) a_{m_{1}}^{\dagger}\right] \cdots\left[R_{i_{p} m_{p}}\left(t, t^{\prime}\right) a_{m_{p}}^{\dagger}\right]\left[R_{i_{i} l_{1}}\left(t, t^{\prime}\right) b_{l_{1}}^{\dagger}\right] \cdots\left[R_{i_{p} l_{p}}\left(t, t^{\prime}\right) b_{l_{p}}^{\dagger}\right]\right| 0\right\rangle \\
& \quad=\Sigma_{i_{1}, \cdots, i_{p}} \int d t^{\prime}\left\langle 0\left|a_{i_{1}} \cdots a_{i_{p}} \cdots b_{i_{1}} \cdots b_{i_{p}}\left\{\mathcal{T} e^{-\int_{t^{\prime}}^{t} \hat{H}\left(t^{\prime \prime}\right) d t^{\prime \prime}}\right\} b_{i_{1}}^{\dagger} \cdots b_{i_{p}}^{\dagger} \cdots a_{i_{1}}^{\dagger} \cdots a_{i_{p}}^{\dagger}\right| 0\right\rangle,
\end{aligned}
$$

where $\hat{H}=\sum_{\alpha \gamma} \Omega^{\alpha \beta} \frac{\partial^{2} \mathcal{H}}{\partial x_{\beta} \partial x_{\gamma}}\left(a_{\alpha}^{\dagger} a_{\gamma}+b_{\alpha}^{\dagger} b_{\gamma}\right)$ generates the linear transformation. In particular,

$$
\begin{aligned}
\operatorname{det} A^{2}(t)= & \Sigma_{i_{1}, \ldots, i_{2 N}} \int d t^{\prime}\langle 0| a_{i_{1}} \cdots a_{i_{2 N}} \cdots b_{i_{1}} \cdots b_{i_{2 N}} \\
& \times\left\{\mathcal{T} e^{-\int_{t^{\prime}}^{t} \hat{H}\left(t^{\prime \prime}\right) d t^{\prime \prime}}\right\} b_{i_{1}}^{\dagger} \cdots b_{i_{2 N}}^{\dagger} \cdots a_{i_{1}}^{\dagger} \cdots a_{i_{2 N}}^{\dagger}|0\rangle .
\end{aligned}
$$

Now, the existence of Lyapunov exponents is the statement that the integral $\mathcal{T} e^{-\int_{t^{\prime}}^{t} \hat{H}\left(t^{\prime \prime}\right) d t^{\prime \prime}} \sim e^{\left(t-t^{\prime}\right) \hat{H}_{o}}$ is extensive to exponential accuracy in time, so that $\mathcal{T} e^{-\int_{t^{\prime}}^{t} \hat{H}\left(t^{\prime \prime}\right) d t^{\prime \prime}} \sim$ $e^{\left(t-t^{\prime}\right) \Lambda^{(p)}}|p\rangle\langle p|$, where $\Lambda^{(p)}$ and $|r\rangle$ are the lowest eigenvalue and corresponding eigenvector of the subspace with $p$ fermions of each kind.

We may thus evaluate the integrals to within exponential accuracy to get

$$
\begin{aligned}
& \operatorname{det} \mathcal{A}^{2}(t) \sim \Sigma_{i_{1}, \ldots, i_{2 N}}\langle 0| a_{i_{1}} \cdots a_{i_{2 N}} \cdots b_{i_{1}} \cdots b_{i_{2 N}}\left(e^{-t \hat{H}_{o}}+1\right) \\
& \quad \times \hat{H}_{o}^{-1} b_{i_{1}}^{\dagger} \cdots b_{i_{2 N}}^{\dagger} \cdots a_{i_{1}}^{\dagger} \cdots a_{i_{2 N}}^{\dagger}|0\rangle .
\end{aligned}
$$

The identity added in the central bracket comes precisely from the times $t^{\prime} \sim t$ and encompasses the effect of the late noise on the contracting modes. We may now distribute the expression for the determinant, which becomes a development in minors:

$$
\operatorname{det} \mathcal{A}^{2}(t) \sim \Sigma_{p} c_{p} e^{t \Lambda^{(p)}}\left[\Lambda^{(p)}\right]^{-1} \propto e^{t \Lambda^{\left(p^{+}\right)}},
$$

where the $c_{p}$ are time-independent overlaps. The sum is dominated by the exponential of the largest of all the exponents, say, $\Lambda^{\left(p^{+}\right)}$, which we hence identify as the KS entropy. Similarly, the expansion of $p$ forms, with $p \leqslant p^{+}$, is dominated by $\Lambda^{(p)}$, so we may identify $\Lambda^{(p)}$ as the sum of the first $p$ Lyapunov exponents. Then, $h_{\mathrm{KS}}=\Lambda^{\left(p^{+}\right)}$, which is precisely the sum of the positive Lyapunov exponents. For a dynamics averaged over a strong noise, it is easy to make this into a rigorous proof.

\section{Classical formula with Poisson brackets: Direct analogy with the quantum case}

We now derive a classical formula which is an analog of Eq. (20). We show how it yields the classical result given in Eq. (17). The way to go from classical to quantum mechanics is

$$
-i[\cdot, \cdot] \rightarrow-\{\cdot, \cdot\}, \quad \operatorname{Tr} \rightarrow \int d x
$$

Thus we expect that for a classical Hamiltonian system, Eq. (20) transforms as

$$
\begin{aligned}
e^{-h_{\mathrm{KS}}^{(2)} t} \propto & \int \mathcal{D} \boldsymbol{\eta} P[\eta] \exp \left(-\frac{1}{2} \sum_{\alpha, \beta} \int d t^{\prime} d t^{\prime \prime} \int d x_{0}\right. \\
& \left.\times \frac{\left\{i\left\{x^{\alpha}\left(t^{\prime}\right), \rho_{0}\left(x_{0}\right)\right\} i\left\{x^{\beta}\left(t^{\prime \prime}\right), \rho_{0}\left(x_{0}\right)\right\}\right\}}{\int d x_{0} \rho_{0}^{2}\left(x_{0}\right)} \eta^{\alpha}\left(t^{\prime}\right) \eta^{\beta}\left(t^{\prime \prime}\right)\right) .
\end{aligned}
$$

Let us now see this in detail.

Classically, we have $\frac{d x}{d t}=-i \mathcal{L} x$, and for the density matrix $\frac{d \rho}{d t}=i \mathcal{L} \rho$, where $-i \mathcal{L} \equiv-\{\cdot, H\}$. The $\mathcal{L}$ superoperator is Hermitian. We can define the following superoperator:

$$
\mathcal{U}\left(t, t^{\prime}\right) f \equiv \mathcal{T} e^{-i \int_{t^{\prime}}^{t} \mathcal{L}\left(t^{\prime \prime}\right) d t^{\prime \prime}} f=\mathcal{T} e^{-\int_{t^{\prime}}^{t}\left\{\cdot, H\left(t^{\prime \prime}\right)\right\} d t^{\prime \prime}} f .
$$

Now, let us turn to our problem. We have the following timedependent Hamiltonian:

$$
H_{h}=H_{0}+h(t) x .
$$

We wish to calculate the Rényi entropy at time $t$, starting with some initial distribution of points in phase space $\rho_{0}$. Thus we look at

$$
\int d x_{t} \rho^{2}\left(x_{t}\right)=\int \mathcal{D} h_{1} \mathcal{D} h_{2} e^{\ln \mathcal{Z}\left(h_{1}, h_{2}\right)}
$$


with

$$
\mathcal{Z}\left(h_{1}, h_{2}\right) \equiv \int d x_{t}\left\{\left[\mathcal{U}_{h_{1}}^{-1}(t, 0) \rho_{0}\right]\left[\mathcal{U}_{h_{2}}^{-1}(t, 0) \rho_{0}\right]\right\}
$$

Expanding to second order around $h_{1}, h_{2}=0$ is easier if we write

$$
\mathcal{U}_{h_{1}}^{-1}(t, 0) \rho_{0}=\mathcal{U}_{h_{1}}^{-1}\left(t, t^{\prime \prime}\right) \mathcal{U}_{h_{1}}^{-1}\left(t^{\prime \prime}, t^{\prime}\right) \mathcal{U}_{h_{1}}^{-1}\left(t^{\prime}, 0\right) \rho_{0} .
$$

Then, for example, the term which is proportional to $h_{1}\left(t^{\prime}\right) h_{1}\left(t^{\prime \prime}\right)$ goes as

$$
\begin{aligned}
a_{1, t^{\prime} ; 1, t^{\prime}} & \propto \int d x_{t} e^{i \mathcal{L}_{0}\left(t-t^{\prime \prime}\right)}\left\{e^{i \mathcal{L}_{0}\left(t^{\prime \prime}-t^{\prime}\right)}\left\{e^{i \mathcal{L}_{0} t^{\prime}} \rho_{0}, x_{0}\right\}, x_{0}\right\} e^{i \mathcal{L}_{0} t} \rho_{0} \\
& =\int d x_{0} e^{-i \mathcal{L}_{0} t^{\prime \prime}}\left\{e^{i \mathcal{L}_{0}\left(t^{\prime \prime}-t^{\prime}\right)}\left\{e^{i \mathcal{L}_{0} t^{\prime}} \rho_{0}, x_{0}\right\}, x_{0}\right\} \rho_{0}=\int d x_{0}\left\{\left\{\rho_{0}, e^{-i \mathcal{L}_{0} t^{\prime}} x_{0}\right\}, e^{-i \mathcal{L}_{0} t^{\prime \prime}} x_{0}\right\} \rho_{0} \\
& =\int d x_{0}\left\{\rho_{0}, e^{-i \mathcal{L}_{0} t^{\prime}} x_{0}\right\}\left\{\rho_{0}, e^{-i \mathcal{L}_{0} t^{\prime \prime}} x_{0}\right\}=\int d x_{0}\left\{\rho_{0}, x\left(t^{\prime}\right)\right\}\left\{\rho_{0}, x\left(t^{\prime \prime}\right)\right\} .
\end{aligned}
$$

For the first equality we use the identity $\int d y f(y) e^{i \mathcal{L} t} g(y)=\int d \tilde{y} e^{-i \mathcal{L} t} f(\tilde{y}) g(\tilde{y})$, where we unitarily change basis $\tilde{y} \rightarrow e^{i \mathcal{L} t} y$; for the second equality we use the identity $\mathcal{U}\{f, g\}=\{\mathcal{U} f, \mathcal{U} g\}$; and for the third equality we use the identity $\int\{f, g\} k=$ $-\int f\{k, g\}$. Therefore, in analogy to the quantum case, we have

$$
a_{1, t^{\prime} ; 1, t^{\prime}} \propto \int d t^{\prime} d t^{\prime \prime} \int d x_{0}\left\{\rho_{0}\left(x_{0}\right), x\left(t^{\prime}\right)\right\}\left\{\rho_{0}\left(x_{0}\right), x\left(t^{\prime \prime}\right)\right\},
$$

and one can conclude that the classical KS entropy follows Eq. (A7). Next, let us show how this alternative classical formula relates to the one derived in the text, Eq. (20).

Let us assume some Gaussian initial condition

$$
\rho_{0}\left(x_{0}\right)=\frac{1}{(2 \pi a)^{d / 2}} e^{-\frac{x_{0}^{2}}{2 a}} .
$$

Plugging this into the argument of the exponent in Eq. (A7), one finds

$$
\int d x_{0} \frac{\left\{x^{\alpha}\left(t^{\prime}\right), \rho_{0}\left(x_{0}\right)\right\}\left\{x^{\beta}\left(t^{\prime \prime}\right), \rho_{0}\left(x_{0}\right)\right\}}{\int d x_{0} \rho_{0}^{2}\left(x_{0}\right)}=\int d x_{0} \frac{\partial x^{\alpha}\left(t^{\prime}\right)}{\partial x_{0}^{\gamma}} \frac{\partial x^{\beta}\left(t^{\prime \prime}\right)}{\partial x_{0}^{\gamma^{\prime}}} \Omega^{\gamma \beta} \Omega^{\gamma^{\prime} \beta^{\prime}} \frac{x_{0}^{\beta} x_{0}^{\beta^{\prime}} \rho_{0}^{2}}{a^{2} \int d x_{0} \rho_{0}^{2}\left(x_{0}\right)} \approx \frac{1}{a} \frac{\partial x^{\alpha}\left(t^{\prime}\right)}{\partial x_{0}^{\gamma}} \frac{\partial x^{\beta}\left(t^{\prime \prime}\right)}{\partial x_{0}^{\gamma}},
$$

where we assumed that $\frac{x_{0}^{\beta} x_{0}^{\beta^{\prime}} \rho_{0}^{2}}{\int \rho_{0}^{2}} \sim a \delta\left(x_{0}^{\beta}-x_{0}^{\beta^{\prime}}\right)$. Equation (A7) then reads

$$
\lim _{\hbar \rightarrow 0} \operatorname{Tr}\left\{\rho^{2}(t)\right\}=(4 \pi a)^{-d / 2} \int \mathcal{D} \boldsymbol{\eta} P[\eta] \exp \left(-\frac{1}{2} \sum_{\alpha, \beta} \int d t^{\prime} d t^{\prime \prime} \frac{R^{\alpha \gamma}\left(t^{\prime}, 0\right) R^{\beta \gamma}\left(t^{\prime \prime}, 0\right)}{a} \eta^{\alpha}\left(t^{\prime}\right) \eta^{\beta}\left(t^{\prime \prime}\right)\right) .
$$

Finally, to solve the Gaussian integral, we can follow the same lines which brought us to Eq. (29), taking the same noise and using the determinant lemma, to find

$$
\ln \operatorname{Tr}\left\{\rho^{2}(t)\right\}=\frac{1}{2} \ln \operatorname{det}\left[1+\frac{\epsilon^{2} \tau}{a} \int d t \mathbf{R}^{T}\left(t^{\prime}, 0\right) \mathbf{R}\left(t^{\prime}, 0\right)\right] .
$$

Then one can repeat the steps indicated after Eq. (40) to see the agreement with the classical result, Eq. (17), in Sec. II.

\section{Semiclassical limit of formula (20)}

Below we show how the semiclassical limit of Eq. (20) (an expression for the quantum KS entropy) gives the classical result of Eq. (A7).

Let us start with Eq. (20). The quantum object which appears in this equation is $\operatorname{Tr}\left(i\left[x^{\alpha}\left(t^{\prime}\right), \rho_{0}\right] i\left[x^{\beta}\left(t^{\prime \prime}\right), \rho_{0}\right]\right)$. We can treat it in the semiclassical limit by introducing the Weyl transform of an operator

$$
\mathcal{W}\{A\}(q, p)=\int e^{-i p y / \hbar}\langle q+y / 2|A| q-y / 2\rangle d y,
$$

which satisfies the two relations: $\operatorname{Tr}(A B)=$ $\int d q d q \mathcal{W}\{A\} \mathcal{W}\{B\}$ and $\mathcal{W}(A) \star \mathcal{W}(B)=\mathcal{W}(A B)$, with $\star$ being the Moyal product. Inserting these into Eq. (20) and taking the semiclassical limit, $f \star g \equiv f g+\frac{i \hbar}{2}\{f, g\}+O\left(\hbar^{2}\right)$, we find

$$
\begin{aligned}
& \frac{\operatorname{Tr}\left(i\left[x^{\alpha}\left(t^{\prime}\right), \rho_{0}\right] i\left[x^{\beta}\left(t^{\prime \prime}\right), \rho_{0}\right]\right)}{\hbar^{2} \operatorname{Tr} \rho_{0}^{2}} \\
& =\frac{\int\left\{x^{\alpha}\left(t^{\prime}\right), \rho_{0}\left(x_{0}\right)\right\}\left\{x^{\beta}\left(t^{\prime \prime}\right), \rho_{0}\left(x_{0}\right)\right\} d x_{0}}{\int \rho_{0}^{2}\left(x_{0}\right) d x_{0}},
\end{aligned}
$$

where it is assumed that $\rho_{0}\left(x_{0}\right)$ and $x^{\alpha}(t)$ are simple functions of $x_{0}$ such that in the semiclassical limit they are given by their Weyl transform [33]. Equation (A19) tells us that the semiclassical limit of Eq. (20) is the classical expression in Eq. (A7). 


\section{APPENDIX B: QUANTUM DERIVATION}

\section{Perturbation theory}

We now provide more details on the derivation of Eq. (20). We shall expand $\ln \operatorname{Tr} \rho^{2}(t)$ in small $\mathbf{h}_{1}(t), \mathbf{h}_{2}(t)$, for the
Hamiltonian $\mathcal{H}=H_{0}(\mathbf{x})+\sum_{\alpha} h^{\alpha}(t) x^{\alpha}$. The density matrix evolves as

$$
\rho(t)=U_{h}(t, 0) \rho_{0} U_{h}^{\dagger}(t, 0),
$$

with $U_{h}(t, 0) \equiv \mathcal{T} \exp \left[-\frac{i}{\hbar} \int \sum_{\alpha} h^{\alpha}\left(t^{\prime}\right) x^{\alpha} d t^{\prime}\right]$. This can be simply seen from taking $\rho_{0}=\left|\phi_{0}\right\rangle\left\langle\phi_{0}\right|$.

Let us consider the derivatives of $\operatorname{Tr} \rho^{2}(t)=\operatorname{Tr}\left[U_{h_{1}}(t, 0) \rho_{0} U_{h_{1}}^{\dagger}(t, 0) U_{h_{2}}(t, 0) \rho_{0} U_{h_{2}}^{\dagger}(t, 0)\right]$. We have that

$$
\frac{\partial \operatorname{Tr} \rho^{2}(t)}{\partial h_{1}^{\alpha}\left(t^{\prime}\right)}=\frac{\partial \operatorname{Tr} \rho^{2}(t)}{\partial h_{2}^{\alpha}\left(t^{\prime}\right)}=0 .
$$

The second derivative consists of several terms:

$$
\begin{aligned}
\hbar^{2} \frac{\partial^{2} \operatorname{Tr} \rho^{2}(t)}{\partial h_{1}^{\alpha}\left(t^{\prime}\right) \partial h_{1}^{\beta}\left(t^{\prime \prime}\right)} & =\hbar^{2} \frac{\partial^{2} \operatorname{Tr} \rho^{2}(t)}{\partial h_{2}^{\alpha}\left(t^{\prime}\right) \partial h_{2}^{\beta}\left(t^{\prime \prime}\right)}=-\hbar^{2} \frac{\partial^{2} \operatorname{Tr} \rho^{2}(t)}{\partial h_{1}^{\alpha}\left(t^{\prime}\right) \partial h_{2}^{\beta}\left(t^{\prime \prime}\right)}=-\hbar^{2} \frac{\partial^{2} \operatorname{Tr} \rho^{2}(t)}{\partial h_{2}^{\alpha}\left(t^{\prime}\right) \partial h_{1}^{\beta}\left(t^{\prime \prime}\right)} \\
& =-\operatorname{Tr}\left\{x^{\alpha}\left(t^{\prime}\right) x^{\beta}\left(t^{\prime \prime}\right) \rho_{0}^{2}+x^{\alpha}\left(t^{\prime}\right) x^{\beta}\left(t^{\prime \prime}\right) \rho_{0}^{2}-x^{\alpha}\left(t^{\prime}\right) \rho_{0} x^{\beta}\left(t^{\prime \prime}\right) \rho_{0}-x^{\beta}\left(t^{\prime \prime}\right) \rho_{0} x^{\alpha}\left(t^{\prime}\right) \rho_{0}\right\} \\
& =\operatorname{Tr}\left\{\left[x^{\alpha}\left(t^{\prime}\right), \rho_{0}\right]\left[x^{\beta}\left(t^{\prime \prime}\right), \rho_{0}\right]\right\}
\end{aligned}
$$

where we define $x(t) \equiv U_{h=0}^{\dagger}(t, 0) \mathbf{x} U_{h=0}(t, 0)=e^{\frac{i}{\hbar} H_{0}(\mathbf{x}) t} \mathbf{x} e^{-\frac{i}{\hbar} H_{0}(\mathbf{x}) t}$.

In summary, we find that

$$
\mathcal{Z}\left[\mathbf{h}_{\mathbf{1}}, \mathbf{h}_{\mathbf{2}}\right] \equiv \ln \operatorname{Tr}\left\{U_{h_{2}}^{\dagger} U_{h_{1}} \rho_{0} U_{h_{1}}^{\dagger} U_{h_{2}} \rho_{0}\right\} \approx \frac{1}{2} \sum_{\alpha, \beta} \int d t^{\prime} d t^{\prime \prime} \frac{\operatorname{Tr}\left\{\left[x^{\alpha}\left(t^{\prime}\right), \rho_{0}\right]\left[x^{\beta}\left(t^{\prime \prime}\right), \rho_{0}\right]\right\}}{\hbar^{2} \operatorname{Tr} \rho_{0}^{2}}\left[h_{1}^{\alpha}\left(t^{\prime}\right)-h_{2}^{\alpha}\left(t^{\prime}\right)\right]\left[h_{1}^{\beta}\left(t^{\prime \prime}\right)-h_{2}^{\beta}\left(t^{\prime \prime}\right)\right],
$$

which gives Eq. (20).

\section{All the Rényi entropies}

We can generalize the derivation to the Rényi entropy of any order. Taking $\rho_{0}=\left|\phi_{0}\right\rangle\left\langle\phi_{0}\right|$, we can write

$$
t h_{\mathrm{KS}}^{(q)}=\ln \operatorname{Tr}\left\{\rho^{q}(t)\right\}=\int \Pi_{r} \mathcal{D} \mathbf{h}_{r} P\left(\mathbf{h}_{r}\right) e^{\sum_{r=1}^{q} \ln \mathcal{Z}\left(\mathbf{h}_{r-1}, \mathbf{h}_{r}\right)},
$$

with $\mathcal{Z}\left(\mathbf{h}_{r-1}, \mathbf{h}_{r}\right) \equiv\left\langle\phi_{0}\left|U_{h_{r-1}, I}^{T} U_{h_{r}, I}\right| \phi_{0}\right\rangle$ and $\mathbf{h}_{0}=\mathbf{h}_{q}$. Expanding in Taylor series,

$$
\sum_{r=1}^{q} \ln \mathcal{Z}\left(\mathbf{h}_{r-1}^{\alpha}, \mathbf{h}_{r}\right) \sim \frac{1}{2} \sum_{r=1}^{q} \sum_{\alpha, \beta} \frac{\partial^{2} \ln \mathcal{Z}}{\partial h_{r-1}^{\alpha} \partial h_{r}^{\beta}} h_{r-1}^{\alpha} h_{r}^{\beta} .
$$

The second derivatives do not depend on $r$ (replica symmetry), so that we obtain a tight-binding problem with $r$ sites. This may be treated as the case of $q=2$ discussed in the main text, and one easily gets

$$
\begin{aligned}
\ln \operatorname{Tr}\left\{\rho^{q}(t)\right\}= & -\frac{1}{2} \sum_{r=1}^{q-1} \ln \operatorname{det}_{\mu, \nu}\left|\delta^{\mu \nu}+\frac{\sin ^{2}\left(\frac{\pi r}{q}\right) \epsilon^{2} \tau}{2 \hbar^{2}} A^{\mu \nu}\right| \\
& -\ln q+(q-1) \ln 2,
\end{aligned}
$$

where the factors $\sin ^{2}\left(\frac{\pi r}{q}\right)$ are the eigenvalues of the matrix with $1 \mathrm{~s}$ in the diagonal and $-\frac{1}{2}$ between nearest neighbors and with periodic boundary conditions. Note that we have not yet averaged over different initial conditions $\rho_{0}$.

All the determinants differ only in a prefactor multiplying $A^{\mu \nu}$, so we conclude that, at least for $q$ of order 1 , there is no multifractality of quantum origin, i.e., given one initial quantum condition. However, of course there will be once we decide to add over different initial conditions, just as there is in the classical case.
[1] A. M. Kaufman, M. E. Tai, A. Lukin, M. Rispoli, R. Schittko, P. M. Preiss, and M. Greiner, Quantum thermalization through entanglement in an isolated many-body system, Science $\mathbf{3 5 3}$, 794 (2016).

[2] W. H. Zurek and J. P. Paz, Decoherence, Chaos, and the Second Law, Phys. Rev. Lett. 72, 2508 (1994).

[3] W. H. Zurek and J. P. Paz, Quantum chaos: A decoherent definition, Physica D 83, 300 (1995).

[4] A. K. Pattanayak, Lyapunov Exponents, Entropy Production, and Decoherence, Phys. Rev. Lett. 83, 4526 (1999).

[5] P. A. Miller and S. Sarkar, Signatures of chaos in the entanglement of two coupled quantum kicked tops, Phys. Rev. E 60, 1542 (1999).
[6] D. Monteoliva and J. P. Paz, Decoherence and the Rate of Entropy Production in Chaotic Quantum Systems, Phys. Rev. Lett. 85, 3373 (2000).

[7] E. Bianchi, L. Hackl, and N. Yokomizo, Entanglement entropy of squeezed vacua on a lattice, Phys. Rev. D 92, 085045 (2015).

[8] C. T. Asplund and D. Berenstein, Entanglement entropy converges to classical entropy around periodic orbits, Ann. Phys. 366, 113 (2016).

[9] A. Hallam, J. G. Morley, and A. G. Green, The Lyapunov spectra of quantum thermalisation, Nat. Commun. 10, 2708 (2019).

[10] A. Connes, H. Narnhofer, and W. Thirring, Dynamical entropy of $C^{*}$ algebras and von Neu- 
mann algebras, Commun. Math. Phys. 112, 691 (1987).

[11] R. Alicki and M. Fannes, Defining quantum dynamical entropy, Lett. Math. Phys. 32, 75 (1994).

[12] H. Gharibyan, M. Hanada, B. Swingle, and M. Tezuka, Quantum Lyapunov spectrum, J. High Energy Phys. 04 (2019) 082.

[13] G. Lindblad, Quantum ergodicity and chaos, in Fundamental Aspects of Quantum Theory, edited by V. Gorini and A. Frigerio (Springer, New York, 1986), p. 199.

[14] P. A. Miller, S. Sarkar, and R. Zarum, Quantum chaos: Entropy signatures, Acta Phys. Pol., B 29, 3643 (1998).

[15] V. Lecomte, C. Appert-Rolland, and F. Van Wijland, Thermodynamic formalism for systems with Markov dynamics, J. Stat. Phys. 127, 51 (2007).

[16] T. Goldfriend and J. Kurchan, Quasi-integrable systems are slow to thermalize but may be good scramblers, Phys. Rev. E 102, 022201 (2020).

[17] F. Ginelli, H. Chaté, R. Livi, and A. Politi, Covariant Lyapunov vectors, J. Phys. A: Math. Theor. 46, 254005 (2013).

[18] P. V. Kuptsov and U. Parlitz, Theory and computation of covariant Lyapunov vectors, J. Nonlinear Sci. 22, 727 (2012).

[19] F. Ginelli, P. Poggi, A. Turchi, H. Chaté, R. Livi, and A. Politi, Characterizing Dynamics with Covariant Lyapunov Vectors, Phys. Rev. Lett. 99, 130601 (2007).

[20] R. Zwanzig, Nonlinear generalized Langevin equations, J. Stat. Phys. 9, 215 (1973).

[21] A. Kamenev, Field Theory of Non-equilibrium Systems (Cambridge University Press, Cambridge, 2011).

[22] L. F. Cugliandolo and G. Lozano, Real-time nonequilibrium dynamics of quantum glassy systems, Phys. Rev. B 59, 915 (1999).

[23] J. Maldacena, S. H. Shenker, and D. Stanford, A bound on chaos, J. High Energy Phys. 08 (2016) 106.

[24] A. Larkin and Y. Ovchinnikov, Quasiclassical method in the theory of superconductivity, Zh. Eksp. Teor. Fiz. 55, 2262 (1969) [Sov. Phys. JETP 28, 1200 (1969)].

[25] R. Fan, P. Zhang, H. Shen, and H. Zhai, Out-of-time-order correlation for many-body localization, Sci. Bull. 62, 707 (2017).

[26] P. Hosur, X.-L. Qi, D. A. Roberts, and B. Yoshida, Chaos in quantum channels, J. High Energy Phys. 02 (2016) 004.

[27] P. D. Bergamasco, G. G. Carlo, and A. M. F. Rivas, Out-oftime ordered correlators, complexity, and entropy in bipartite systems, Phys. Rev. Research 1, 033044 (2019).

[28] E. B. Rozenbaum, S. Ganeshan, and V. Galitski, Universal level statistics of the out-of-time-ordered operator, Phys. Rev. B 100, 035112 (2019).

[29] A. Perelomov, Generalized Coherent States and Their Applications (Springer, New York, 2012).

[30] G. Paladin and A. Vulpiani, Intermittency in chaotic systems and Renyi entropies, J. Phys. A: Math. Gen. 19, L997 (1986).

[31] E. Gozzi and M. Reuter, Lyapunov exponents, path-integrals and forms, Chaos, Solitons Fractals 4, 1117 (1994).

[32] S. Tanase-Nicola and J. Kurchan, Statistical-mechanical formulation of Lyapunov exponents, J. Phys. A: Math. Gen. 36, 10299 (2003).

[33] W. B. Case, Wigner functions and Weyl transforms for pedestrians, Am. J. Phys. 76, 937 (2008). 